\title{
Self-Reference and Chaos in Fuzzy Logic
}

\author{
Patrick Grim
}

\begin{abstract}
The purpose of this paper is to open for investigation a range of phenomena familiar from dynamical systems or chaos theory which appear in a simple fuzzy logic with the introduction of self-reference. Within that logic, self-referential sentences exhibit properties of fixed point attractors, fixed point repellers, and full chaos on the $[0,1]$ interval. Strange attractors and fractals appear in two dimensions in the graphing of pairs of mutually referential sentences and appear in three dimensions in the graphing of mutually referential triples.
\end{abstract}

\section{INTRODUCTION}

C HAOS theory and fuzzy logics form two of themost intriguing and promising areas of current mathematical research. In what follows, I want to explore a region of fuzzy logic which exhibits some of the phenomena of chaos theorya region found, interestingly enough, in a consideration of the fuzzy dynamical semantics of self-referential paradoxes and related sentences.

A familiar fuzzy logic is outlined in Section I. In section II, the classical example of the Liar paradox is used to sketch the use of iterated algorithms to model self-reference. Self referential sentences in fuzzy logic displaying the dynamical semantics of fixed-point attractors and fixed-point repellers are then outlined in Section III. Section IV is devoted to the Chaotic Liar, a fuzzy self-referential sentence the dynamical semantics of which is fully chaotic in the precise mathematical sense. Section $V$ traces similar results with regard to pairs of mutually referential sentences, introducing strange attractors and fractals in two dimensions. In Section VI, the exploration is extended to triples of mutually referential sentences and corresponding dynamical phenomena in three dimensions.

Here, my purpose is merely to call attention to some of the dynamic phenomena of self-referential fuzzy logics, and I will proceed for the most part by simple example. In the somewhat different context of [1], a hope was expressed that a next step in research would reveal fuzzy systems with chaotic behavior "and then we can define, and study, fuzzy fractals" [2], [3].

The work that follows seems to fulfill that hope, here inthe context of a study of the dynamical semantics of self-reference within fuzzy logic.

Given the affinity of many of the examples that follow to the traditional semantic paradoxes, it should perhaps be emphasized that this paper is not to be construed as an attempt to solve the paradoxes. Two thousand years of attempted solutions can hardly be said to have met with conspicuous success, and I do not expect fuzzy logics to be any less

Manuscript received January 16, 1992; revised May 11, 1993

The author is with the Group for Logic and Formal Semantics, Department of Philosophy, SUNY at Stony Brook, Stony Brook, NY.

IEEE Log Number 9213142. vulnerable to strengthened versions of the Liar, say, than are three-valued, multivalued, infinite-valued, gapped, and antifoundational logics (for a critical survey, see for example [4, ch. 1]). In the end, a solution to the phenomena of selfreference may simply be the wrong thing to look for. In something of the spirit of [5]-[7], the attempt here is rather to open for investigation the semantical dynamics of selfreference and self referential reasoning in their own right, here within a fuzzy logic and with an emphasis on patterns of semantic instability.

Although I attempt at various points to draw some speculative conclusions, it is clear that much remains to be done in terms of generalization and interpretation. Intriguing and perhaps even beautiful formal phenomena appear in the semantics of certain self-referential sentences within fuzzy logic, but it is not always clear why they appear, how they generalize, or what such formal phenomena actually mean. It is known that there are a range of metamathematical results closely related to aspects of the current work, including extensions of classical limitative results to both chaos theory and fuzzy logic [8], [9]. I leave a more complete discussion of those topics, however, to another paper.

\section{A Simple FuZZY LOGIC}

The basic fuzzy logic to be used in what follows is perhaps thesimplest possible: the familiar Lukasiewicz $\mathrm{L}_{\aleph 1}$. Our numerical truth values are taken to be the reals in the $[0,1]$ interval, using as connectives:

$$
\begin{aligned}
\underline{v}(\sim p)= & 1-\underline{v}(p) \\
\underline{v}(p \wedge q)= & \min [\underline{v}(p), \underline{v}(q)] \\
\underline{v}(p \vee q)= & \max [\underline{v}(p), \underline{v}(q)] \\
\underline{v}(p \rightarrow q)= & \min [1,1-\underline{v}(p)+\underline{v}(q)] \\
\underline{v}(p \leftrightarrow q)= & \underline{v}((p \rightarrow q) \wedge(q \rightarrow p)) \\
= & \min [\min [1,1-\underline{v}(p)+\underline{v}(q)], \\
& \min [1,1-\underline{v}(q)+\underline{v}(p)]] \\
= & 1-\operatorname{abs}(\underline{v}(p)-\underline{v}(q))
\end{aligned}
$$

where $\underline{v}(p)$ denotes the numerical truth value of a proposition $p$ [10], [11].

Formal considerations cast a strong presumption in favor of minand max for conjunction and disjunction [12], and an only slightly weaker presumption in favor of the treatment of negation employed here. The same cannot be said for the Lukasiewicz implication: here, it must simply be admitted that 
there are a number of alternatives. ${ }^{1}$ Nonetheless, it is clear that $\mathrm{L}_{\mathrm{N} 1}$ can currently claim to be the standard basis for fuzzy logic.

The distinguishing mark of true fuzzy logics, as opposed to mere infinite-valued logics, is the use of a denumerable set of "linguistic" truth values beyond the numerical truth values $\underline{v}(p)$ used above. ${ }^{2}$ Linguistic truth values are themselves represented by fuzzy sets, standardly generated from a fuzzy set "true" and its converse "false" through recursive application of algorithmic hedges such as "very," "more or less," "slight," and the like [11], [15]-[19].

Nothing in the basic Lukasiewicz logic, however, dictates what shape a basic fuzzy set for "true" is to take. Here, as in the case of implication, there are clearly alternatives. For present purposes, I want to use what may be the simplest and most clearly justified fuzzy set for "true," generated by importing the familiar Tarski convention $T$ directly into $L_{\$ 1}$.

Following [20], and using $T(p)$ for the claim that " $p$ " is true,

$$
T(p) \leftrightarrow p .
$$

Using the Lukasiewicz biconditional,

$$
\begin{aligned}
\underline{v}(T(p) \leftrightarrow p) & =\underline{v}((T(p) \rightarrow p) \wedge(p \rightarrow T(p)) \\
& =1-\operatorname{abs}(\underline{v}(T(p))-\underline{v}(p)) .
\end{aligned}
$$

Assuming that the Tarskian schema itself takes the value of " 1 ," for absolute truth, it must be the case that:

$$
\underline{v}(T(p))=\underline{v}(p)
$$

or, for those more familiar with Zadeh's symbolism,

$$
\mu_{\text {true }}(\underline{v})=\underline{v} .
$$

What direct importation of the Tarskian $T$ schema into $\mathrm{L}_{\aleph 1}$ gives us is the basic fuzzy set for "true" of [17], [18], [21], [22]. With "false" as the complement of "true" and modeling "very" and "fairly" or "more or less" in terms of squares and square roots, respectively-a treatment fairly consistent across the literature-we get the basic set of linguistic truth values indicated in Fig. 1.3

This treatment of "true" does, of course, have alternativesmost notably that of [11], in which a fuzzy set for "true" is characterized as:

$$
\begin{aligned}
\mu_{\text {true }}(\underline{v}) & =0 \quad \text { for } 0 \leq \underline{v} \leq \alpha \\
& =2\left(\frac{\underline{v}-\alpha}{1-\alpha}\right)^{2} \quad \text { for } \alpha \leq \underline{v} \leq \frac{\alpha+1}{2} \\
& =1-2\left(\frac{\underline{v}-1}{1-\alpha}\right)^{2} \quad \text { for } \frac{\alpha+1}{2} \leq \underline{v} \leq 1
\end{aligned}
$$

In [8], for example, we use an implication definable as $(\sim p \vee q)$, which gives us Rescher's $S_{\aleph}^{\supset}$ [10], first developed in [13]. As [14] notes, this approach offers a direct fuzzification of predicate calculus.

It should also be noted that a stronger intuitive argument might be made for the Lukasiewicz biconditional than for the conditional itself. It is the biconditional rather than the conditional that is most directly relied on in what follows.

${ }^{2}$ See [14] on different senses of "fuzzy logic."

${ }^{3}$ Here, I do not mean to suggest that the standard modeling for "very" and "fairly" is any way beyond question, of course. On this, see the discussions in Sections IV and footnote 17.

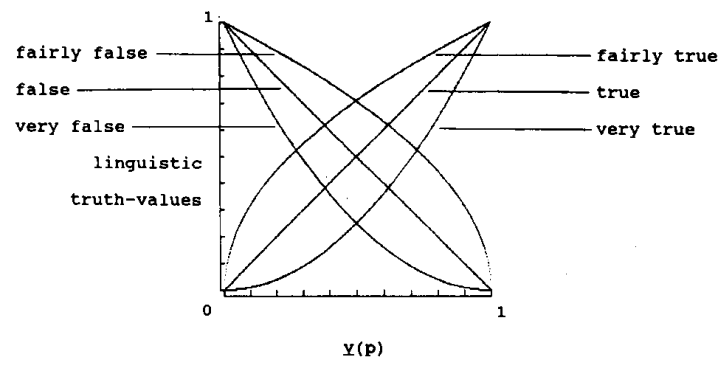

Fig. 1.

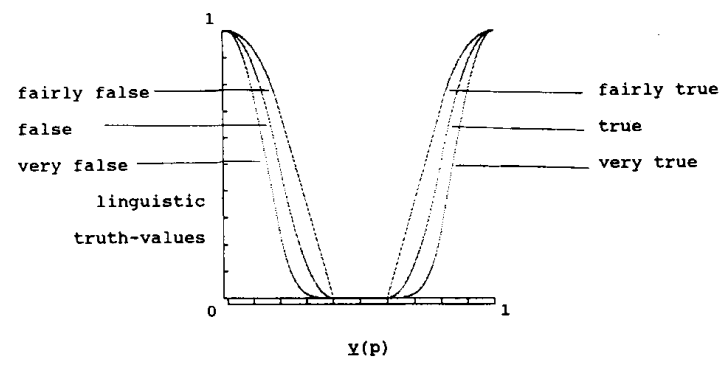

Fig. 2.

where $\alpha$ is a parameter "which indicates the subjective judgment about the minimum value of $\underline{v}$ in order to consider a statement as "true" at all" [19]. A sketch of Zadeh's basic fuzzy sets for "true" and "false" (where $\mu_{\text {false }}(\underline{v})=\mu_{\text {true }}(1-$ $\underline{v})$ ), using an $\alpha$ of 0.6 along with the corresponding sets for "very" and "fairly," appears in Fig. 2.

The results of this paper are built on Baldwin's fuzzy logic rather than Zadeh's for reasons of simplicity: Baldwin's outline for "true" is simpler not only in algorithmic and graphic terms but in terms of its justification as a direct importation of the Tarski $T$-schema into Lukasiewicz $\mathrm{L}_{\aleph_{1}}$. It is clear that a range of results similar to those described later, however, would emerge from a consideration of Zadeh's logic or other more complicated fuzzy logics as well; at several points results are indicated which will hold for any fuzzy logic with $\biguplus_{\aleph 1}$ as a base.

It is important to note that although the underlying semantics (or model) of our logic is expressed in terms of numerical truth values, the propositions admissable in the language of the logic itself can use only linguistic truth values, " $p$ is very true" is, thus, a type of sentence for which our fuzzy logic provides a valuation scheme; " $p$ is 0.75 true" is not. The perceived artificiality of sentences of the latter sort does, in fact, seem to be have been part of Zadeh's initial motivation for moving to linguistic truth values [11], and much of the common suspicion of the "artificial numbers" of fuzzy logics can be dealt with by viewing those numbers merely as an artifact of the semantic model. The language for which that model is designed need not contain numerical truth values at all. ${ }^{4}$

\footnotetext{
${ }^{4}$ Here, the case of set-theoretical semantics is perfectly parallel. The fact that our semantic model is written in terms of sets need not commit us to thinking that the sentences modeled are themselves about sets at all.
} 
Consider also a language, however, in which we are allowed to claim numerical truth values for particular propositions--" $p$ is 0.75 true"--just as we normally do within the semantics of fuzzy logic. Suppose, moreover, that we have a claim $q$ that we know to be 0.75 true. In that case, the claim that " $p$ is 0.75 true" will intuitively be as true as the claim that $p$ has the same value as $q$. For this particular case, and using the biconditional quite naturally to represent "has the same value as," we can express that basic intuition as:

$$
p \text { is } 0.75 \text { true } \leftrightarrow(p \leftrightarrow q) \text {. }
$$

In general, let $[v]$ represent a proposition with fixed numerical value $v$. By the same reasoning, the claim that " $p$ is $v$ true" will be as true as the claim that $p$ has the same value as $[v]$. This we can express in terms of a biconditional as:

$$
p \text { is } v \text { true } \leftrightarrow(p \leftrightarrow[v])
$$

If this general intuitive principle is itself thought of as having a value of 1 , the Lukasiewicz biconditional gives us:

$$
\underline{v}(p \text { is } v \text { true })=1-\operatorname{abs}(\underline{v}(p)-\underline{v}([v]))
$$

or simply

$$
=1-\operatorname{abs}(\underline{v}(p)-v) .
$$

This, it turns out, is Rescher's truth value assignment operator for infinite-valued logic [10]. In terms of Rescher's schema, the value for a proposition $V v p$ to the effect that a proposition $p$ has a numerical truth value $v$ is given by:

$$
\underline{v}(V v p)=1-\operatorname{abs}(v-\underline{v}(p)) .
$$

This relation between Baldwin's fuzzy sets for linguistic truth values and Rescher's $V v p$ schema for attributions of numerical truth values is perhaps even clearer when envisaged graphically. With numerical values on axes, the graph for a proposition " $V 1 p$ " to the effect that a proposition $p$ has value 1, given Rescher's treatment, is precisely Baldwin's graph for " $p$ is true" in Fig. 1. The Rescher graph for " $V 0 p$ " corresponds to Baldwin's graph for " $p$ is false."

The infinite-valued Rescher scheme is central to [8], [9], [23]. Here, I want to focus on dynamical semantic phenomena within the more strictly fuzzy logic outlined.

\section{SELF-REFERENCE AS ItERATION: THE EXAMPLE OF THE LIAR}

Consider, for a moment, the Liar sentence in the context of a classical logic:

$$
\text { This sentence is false. }
$$

If true, (1) must be false. If false, since the Liar says it is false, it must be true. When one first approaches the Liar, one is forced into an intuitive pattern of reasoning that seems to oscillate in conclusion between "true" and "false:" if true, the Liar must be false ... but then if false, it must be true... but then if true, it must be false: ${ }^{5}$

$$
T, F, T, F, T, F, \cdots \text {. }
$$

In these familiar intuitive terms, this alternation is quite literally a temporal one: one is first drawn to the conclusion that the Liar must be true, later forced to the conclusion that it must be false, and so forth. It is also the case that finite reasoners such as ourselves are generally smart enoughor logically unprincipled enough, or both-to break out of such a series rather than to continue it indefinitely. In what follows, however, I wish to abstract from both of these points. The dynamical semantics of a sentence such as the Liar can be thought of as a series of revised semantic values forced by something like the standard Liar argument. As such, the points of oscillation represent not so much discrete times as discrete abstract steps in a pattern of protracted reasoning. ${ }^{6}$ If we think of these as values arrived at by a reasoner, that reasoner should be thought of as an idealized reasoner acting without time constraints and purely on logical principle. $^{7}$

\footnotetext{
${ }^{5}$ Semantic paradox has had, of course, a long and distinguished career in philosophical and mathematical logic. It lies at the core of Cantor's diagonal argument and the paradise of transfinite infinities it offers. Russell's paradox, discovered as a simplification of Cantor's argument, was historically instrumental in motivating axiomatic set theory. Gödel [24] explicitly uses the Liar paradox (and a relative known as the Richard paradox) to motivate his incompleteness theorems, and the limitative theorems of [20], [25], [26] can all be seen as exploiting the basic reasoning of the Liar. In the mid-1960's, Chaitin [27] developed an interpretation of Gödel's theorem in terms of the notion of algorithmic randomness by formalizing the Berry paradox, itself a simplication of the Richard.

In recent years, philosophers have repeatedly attempted to find solutions to the semantic paradoxes by seeking patterns of semantic stability--hence, the proliferation of "truth-value gap solutions" of the 1960's and 1970's [28]-[30] Efforts in the direction of finding patterns of stability within the paradoxes continued in the 1980's with the work of H. Herzberger [5] and A. Gupta [6]. Recent work in this vein also includes that of J. Barwise and J. Etchemendy [31], using Aczel set theory with an antifoundation axiom to characterize [31], using Aczel
Liar-like cycles.

The work of this paper, in contrast, like that of [8], [9], can be seen as an attempt to study complex patterns of instability in the general domain of self-reference and paradox.

${ }^{6}$ In [32, p. 218], dynamical systems are spoken of intuitively as the description of the time behavior of a point moving about on some sor of surface according to a rule that describes how one point is to follow another. Here, as in other contexts, I think that restriction to time cannot be taken too literally. Within ecological studies, iterative "times" may in fact be generations; within epidemiological studies, "times" may be (variable) periods of vulnerability to infection and, of course, the pure mathematics of iterated functions need not be thought of in terms of literal time at all.

Many attempts to solve the Liar and similar paradoxes, of course, rely on denying that there is a genuine oscillation here by insisting that, at each step, one is using a distinct truth predicate or has ascended to a higher metalanguage. That move itself, however, is quite clearly counterintuitive. (As Barwise and Etchemendy note. "When we think about the Liar on an intuitive level, there is an inclination to claim that the truth value "flips back and forth." First, we see that it is false, then that it is true, then that it is false, and so forth." [31, p. 136]). Here and throughout, as noted in the Introduction, my attempt (like that of [5]-[7]) is to track the intuitive dynamical semantics of self-referential sentences rather than to sacrifice semantic intuitions in a too-quick search for a "solution" to the paradoxes.

${ }^{7}$ The idealizations and abstractions at issue clearly make our semantic model "objectivist" in spirit. For a critique of objectivist approaches, in general, see [33, esp. $205 \mathrm{ff}$.]
} 


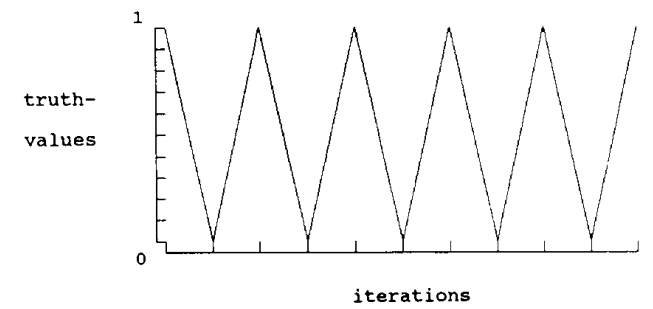

Fig. 3.

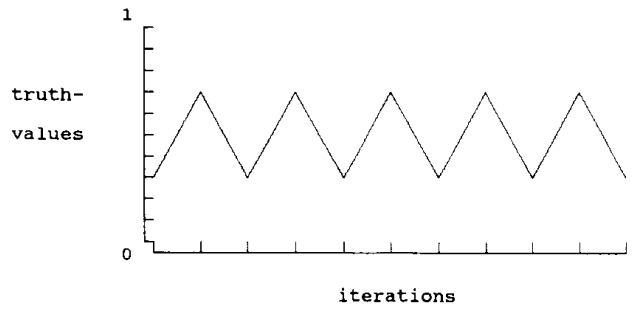

Fig. 4.

Using 1 for truth and 0 for falsity, we can then model the classical semantic behavior of the Liar in terms of a sequence of values $x_{n}$, where $x_{0}$ is an initial or "seed" value and

$$
x_{n+1}=1-x_{n}
$$

With a seed value of 1 , the dynamic semantics of the Liar within a classical logic can, thus, be graphed as in Fig. 3 .

A seed value of 0 , of course, shows an identical oscillationshifted one iteration to the left. In what follows, iterated algorithms of this type will be used to model the dynamical semantics of self-referential sentences quite generally.

As a first example, consider the Liar sentence within the context of the fuzzy logic outlined--a variation we might term the Fuzzy Liar. Given the Baldwin fuzzy set for "false," our algorithm remains the same, though now of course we need to consider numerical truth values in the full $[0,1]$ interval.

For a seed value of 0.3 , the Fuzzy Liar gives an oscillation between 0.3 and 0.7 (see Fig. 4).

For any seed value $x$, in fact, the dynamical semantics of the Fuzzy Liar will be a simple oscillation between $x$ and $(1-x)$. The one fixed point is 0.5 .

Often, the dynamical semantics of self-referential sentences is better,illustrated using a web diagram. In a graph such as that in Fig. 5, we start with a plotted function for, say, "false." An initial seed value $a(0.3$, in this case $)$ is plotted as $(a, 0)$, and a line drawn from that point vertically to the function $f(x)$ [Fig. 5(a)]. We read off our $y$ value as indicating that $f(x)$ for $x=0.3$ is 0.7 . In order to represent the iteration of that function, we now draw a horizontal line to a point $[f(a), f(a)]$ on the diagonal $x=y$-thereby converting our previous $y$ value to a new $x$ value--and then draw a vertical line again from that point to our function at $[f(a), f(f(a))]$
IEEE TRANSACTIONS ON FUZZY SYSTEMS, VOL. 1, NO. 4, NOVEMBER 1993

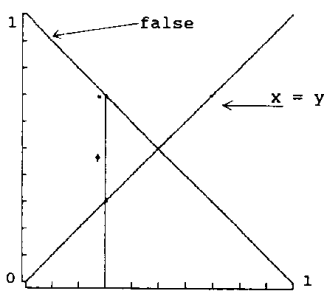

(a)

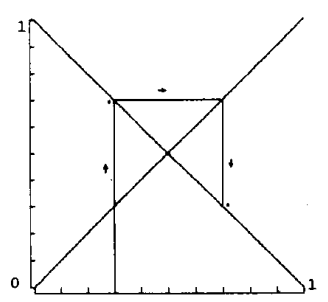

(b)
Fig. 5 .

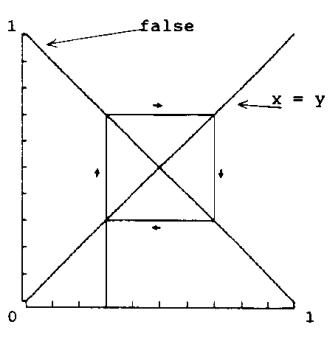

the Fuzzy Liar for .3

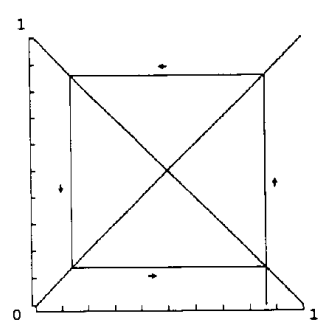

the Fuzzy Liar for .86
Fig. 6.

[Fig. 5(b)]. The $y$ value of this intersection point indicates that $f(x)$ for $x=0.7$ is 0.3 [Fig. 5(b)]. We graph the results of repeated iteration by continuing the process, at each step converting our $y$ value to an $x$ value by reflecting off the $x=y$ diagonal, giving us a new point of intersection with our plotted function.

Within such a web diagram, it is clear that a seed value of 0.3 for Fuzzy Liar will give us a simple box, representing the period two oscillation between 0.3 and 0.7 . A seed value of 0.86 gives us a broader box (Fig. 6).

Because the Fuzzy Liar sets up an oscillational dynamical semantics of this type, statements which are not self-referential but which attribute some linguistic truth value to the Fuzzy Liar will have semantics with the same pattern. In this sense, the dynamical semantics of the Fuzzy Liar prove contagious.

Consider, for example, the following fuzzy statement about the fuzzy Liar:

The Fuzzy Liar is very true

or the second satement of the pair:

This sentence is false.

(1) is very true.

For a seed value of 0.3 , we have seen the Fuzzy Liar alternate between 0.3 and 0.7 . Using the standard squaring function for "very true," the value of (2) will then alternate between 0.09 and 0.49 . For a seed value of 0.54 , to use another example, the 


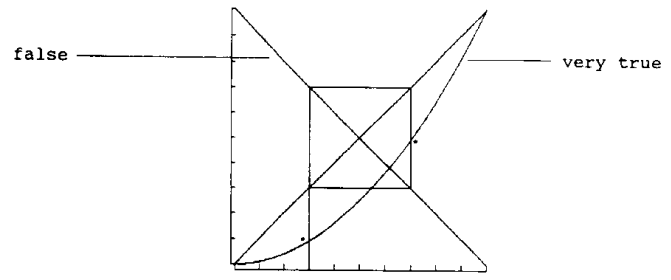

Fig. 7.
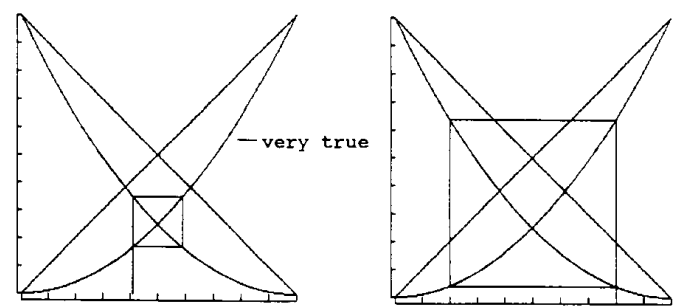

Fig. 8 .

Fuzzy Liar alternates between 0.54 and 0.46 . In that case, (2) will correspondingly alternate between 0.2916 and $0.2116^{8}$

The derivative semantic behavior of (2) can be thought of in terms of a web diagram as follows. Let us start with an initial seed value of 0.3 for the Fuzzy Liar, represented on the $x$ axis as $(0.3,0)$. If $(1)$, thus, has a value of $0.3,(2)$ will have a value of 0.09 , reflected by the fact that a line drawn vertically from $(0.3,0)$ intersects our function for "very true" at $(0.3,0.09)$. Given a seed value of 0.3 , however, we will also be forced to a revised value for the Fuzzy Liar of 0.7 , reflected by the fact that our vertical line intersects the function for the Fuzzy Liar at $(0.3,0.7)$ (see Fig. 7).

Here, we are interested in successive values for (2). In order to obtain the next value for (2), however, we cannot simply reflect $(0.3,0.09)$ off the $x=y$ diagonal as before. We have instead to work from the revised value 0.7 for the Fuzzy Liar, reflect that off the $x=y$ diagonal, and then drop a line vertically to again intersect our "very true" function for (2).

For reasons discussed at a slightly later point in the paper, we can also graph progressive values for (2) directly by reflecting the $y$ value of our earlier point $(0.3,0.09)$ off not the $x=y$ diagonal but the mirror image left to right of our graph for (2).

${ }^{8}$ On the pattern of the Liar, the intuitive reasoning here will proceed something as follows. If we assume that (1) has a value of 0.3 , then (2) will be fairly false with a value of 0.09 . On the assumption that (1) has a value of 0.3 , however, since (1) says it is false, (1) will be fairly true--it will have a value of 0.7 . However, (2) will not be so far off after all, receiving a value of 0.49 . If (1) has a value of $0.7 \cdots$. Despite this alternation, the spirit of Zadeh's extension principle $[11,416 \mathrm{ff}$.]) appears to be preserved at each step.

The intuition that we should, nonetheless, be able to say something constant about (1)'s truth value, despite its oscillation, can perhaps be addressed only in a language in which we explicitly introduce predicates such as "is not consistently true" or "has no constant truth value." In this paper, I have concentrated on chaotic relatives of the Liar; in that stronger language, I believe we should expect chaotic relatives of the Strengthened Liar.

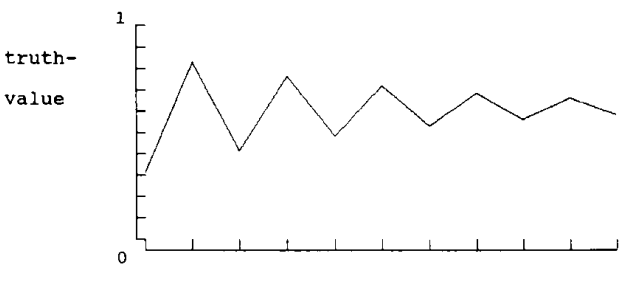

iterations

Fig. 9.

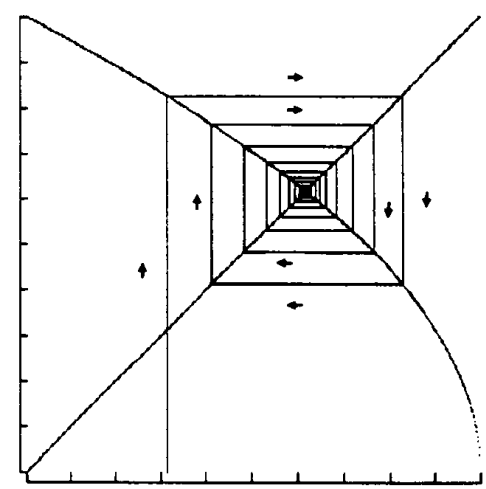

Fig. 10 .

An initial value of 0.41 for the Fuzzy Liar gives us a graph for (2) shown on the left in Fig. 8. An initial value of 0.8 gives us the graph on the right.

In each case, our graph again shows a box, though here in a different position, reflecting the fact that semantic values for sentences which attribute truth values to the Fuzzy Liar will oscillate in the same way that semantic values for the Fuzzy Liar itself do.

\section{ATtRaCtOR AND REPELlER FIXED POINTS IN THE PHENOMENA OF SELF-REFERENCE}

To this point, we have concentrated on the simple Liar within fuzzy logic. The same basic techniques also allow us to model a wider spectrum of self-referential sentences with a wider class of dynamic semantic behaviors.

Consider, for example,

This sentence is fairly false.

I will refer to (3) as the Modest Liar. In terms of our basic logic, the dynamical semantics of (3) can be modeled using the following algorithm:

$$
x_{n+1}=\sqrt{1-x_{n}} .
$$

In a simple bounce diagram, for a seed value of 0.314 , this gives us Fig. 9:

The semantical behavior of the Modest Liar is still clearer, however, in a web digram (Fig. 10). 


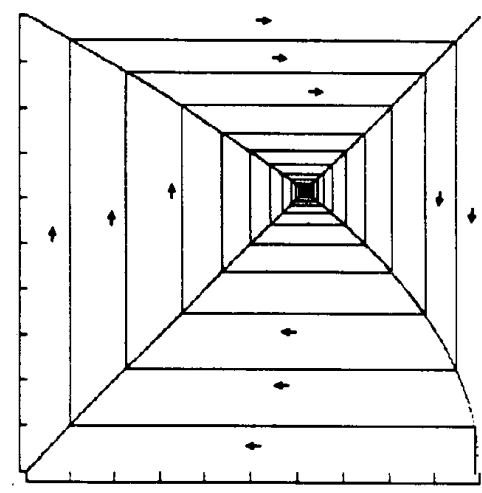

Fig. 11 .

For any seed value, it turns out, the Modest Liar converges inexorably on a fixed point attractor of $(-1+\sqrt{5}) / 2 .{ }^{9} \mathrm{Fig} .11$ shows the Modest Liar with a seed value of 0.99 .

The fact that the Modest Liar has a semantics with a fixed point attractor of this sort means that it is, in a sense, a selfreferential limiting case with regard to fuzziness. Whatever element of a fuzzy range of numerical values we might assign to such a sentence initially, repeated calculation will force us to a very precise and single value: the Modest Liar seems to convert fuzziness to precision.

It is clear that the precise behavior of the Modest Liar relies on the use of a square root function to model "fairly." Although that use is fairly consistent across the literature, as noted, it is also clearly open to challenge: why insist on square root in particular? The use of $\sqrt[3]{,}, 4$, or the like would give us semantic behavior similar to that of the Modest Liar, though converging on a different fixed point. Here, I leave as open questions what the fixed point would be for an arbitrary $n$ root and whether that has anything to tell us about the appropriateness or inappropriateness of different roots in modeling hedges such as "fairly."

Consider also an Emphatic Liar:

This sentence is very false.

Semantic values for the Emphatic Liar will be determined by:

$$
x_{n+1}=\left(1-x_{n}\right)^{2} .
$$

For a seed value of 0.3 , the Emphatic Liar forces a series of revised values which eventually converge on the familiar oscillation between 0 and 1 characteristic of the classical Liar (see Fig. 12).

With one exception, the Emphatic Liar will force any numerical value in the $[0,1]$ interval to the oscillation of

\footnotetext{
${ }^{9}$ The solution to $x=\sqrt{1-x}$ is $(-1 \pm \sqrt{5}) / 2$. Only $(-1 \pm \sqrt{5}) / 2$ appears within our semantic interval $[0,1]$, however. Similar comments apply with respect to $x=(1-x)^{2}$ and the semantic fixed point $(3-\sqrt{5}) / 2$ for the Emphatic Liar. In an entertaining knights-and-knaves exploration of some of these ideas, Hellerstein [34] refers to the Modest Liar as the Golden Liar, pointing out that its attractor fixed point is $1 / \phi$, where $O$ is the golden ratio. The repeller fixed point for the Emphatic Liar is similarly $1-1 / \phi$. The golden ratio $\phi$ itself turns up in a number of surprising places: $\phi$ is the limit of the Fibonacci series $1 / 1,2 / 1,3 / 2,5 / 3,8 / 5 \cdots ; \phi-1=1 / \phi$; $\phi=\sqrt{1+\sqrt{1+\sqrt{1+}}} \cdots ;$ etc. See $[35$, pp. 203-206].
}

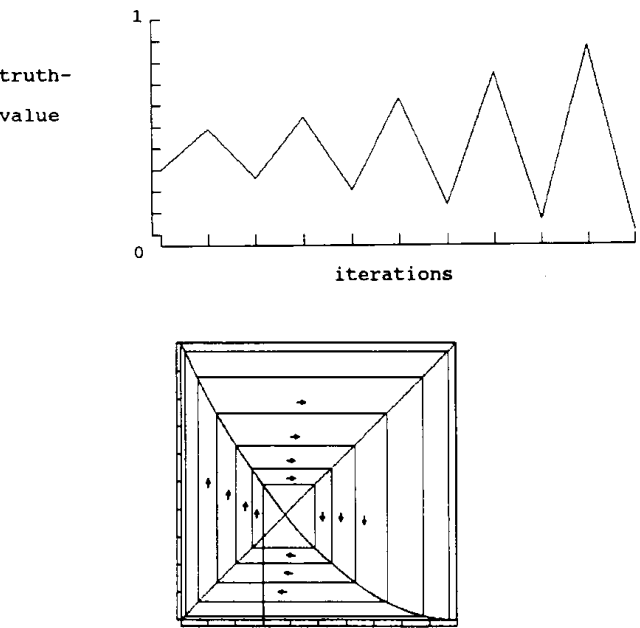

Fig. 12

the classical Liar: the semantical dynamics of the Emphatic Liar is that of a fixed repeller point at $(3-\sqrt{5}) / 2$. The one point that is not forced out to the behavior of the classical Liar is the point $(3-\sqrt{5}) / 2$ itself. The Emphatic Liar can, thus, be seen as a self-referential limiting case to fuzziness in much the same way as can the Modest Liar. The Modest Liar, however, forces convergence on a precise nonclassical value. With one exception, the Emphatic Liar forces revised values to a Liar-like oscillation between the two classical values of 0 and $1 .^{10}$

The use of squaring to model "very" is, of course, open to question in ways similar to those indicated regarding the use of square root for "fairly." An Emphatic Liar using $\left(1-x_{n}\right)^{3}$ or $\left(1-x_{n}\right)^{4}$ would still converge to a Liar-like oscillation, though from a different repeller point. Here, again it remains unclear whether this has anything to tell us about the proper modeling of hedges such as "very" or "fairly."

What of statements which attribute fuzzy truth values to sentences such as the Modest and Emphatic Liars? Here, as in the case of the Fuzzy Liar, we will have sentences with a semantics dependent on that of the Liars themselves. Consider, for example,

The Modest Liar is very false

or (6):

This sentence is fairly false.

(3) is very false.

${ }^{10}$ Hellerstein has also suggested an Equivocal Liar:

This sentence is not very true

with an algorithm

$$
x_{n+1}=1-x_{n}^{2}
$$

Here, $(-1+\sqrt{5}) / 2$ is a repelling fixed point, forcing values out to an oscillation between 0 and 1 . 


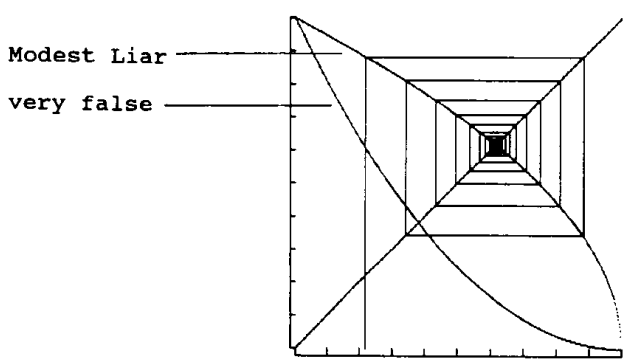

Fig. 13.

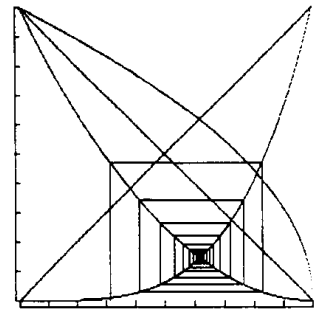

seed: .314

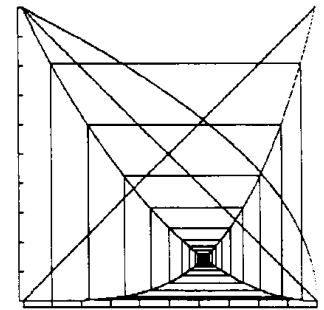

seed: .1
Fig. 14.

For a seed value of 0.3 , the Modest Liar gives us the following series, converging as we have seen on $(-1 \pm \sqrt{5}) / 2$ :

$$
0.3,0.83667,0.40415,0.77191, \ldots 11
$$

If the Modest Liar has a value of 0.3 , however, the claim that it is very false takes a value of $(1-0.3)^{2}$ or 0.49 . Given a revised value of 0.83667 for the Modest Liar, we are forced to revise the value of (6) accordingly, to 0.02668 . Thus, the progressive dynamics of the Modest Liar force a corresponding dynamics for (6):

$$
0.49,0.02668,0.35503,0.05202, \cdots \text {. }
$$

This pattern of dependent dynamical semantics can be illustrated in a web diagram as follows. In Fig. 13, we have graphed functions for both the Modest Liar and "very false." For a given seed value $x$, we draw a line vertically for $(x, 0)$ to intersect the Modest Liar function. The $y$ value of this point of intersection $(x, y)$ is, of course, our revised numerical truth value for the Modest Liar, and reflection off the $x=y$ line will give us the next value for the Modest Liar.

At each step, the value of (6) depends on that of the Modest Liar and is, in fact, the value at which our line representing $x$-value intersects the function line for (6). Thus, we can think of progressive values for $(6)$ as intersection points on the "very false" curve directly below the progressive points on the graph for the Modest Liar.

In this case, we can also graph the dynamics of the dependent sentence (6) more directly, however, by reflecting its values off the $x^{4}$ curve. Progressive values for (6) starting with two different seed values for (3), appears as in Fig. 14.

${ }^{11}$ Here, I exhibit only approximate values, rounded off for the sake of simplicity.
Not surprisingly, attribution of fuzzy linguistic truth values to the Modest Liar shows a convergence to the same fixed point as does the Modest Liar itself.

It is perhaps worth noting why $x^{4}$ is used as a reflectioncurve in this case. So as to avoid confusion with $x$ and $y$ values, let us express the algorithms at issue in terms of variables $a$ and $b$ :

This sentence is fairly false

$a_{n+1}=\sqrt{1-a_{n}}$

(3) is very false

$$
b_{n}=\left(1-a_{n}\right)^{2}
$$

For a given value $b_{n}$ for (6), the calculation of the next value $b_{n+1}$ can be thought of as proceeding in several steps.

$1-\sqrt{b_{n}}$ We first use the inverse of our algorithm for (6) for the case of $0 \leq b_{n} \leq 1$ in order to give us $a_{n}$.

$\sqrt{1-\left(1-\sqrt{b_{n}}\right)}$ By embedding this result in the algorithm for (3), we then obtain the value $a_{n+1}$.

$\left(1-\left(\sqrt{1-\left(1-\sqrt{b_{n}}\right)}\right)\right)$ By embedding this result in the algorithm for (6), we obtain $b_{n+1}$.

The calculation is captured in a reflective graph as follows. For $0 \leq b_{n} \leq 1,\left(\sqrt{1-\left(1-\sqrt{b_{n}}\right)}\right)$ amounts to $\sqrt[4]{b_{n}}$. For the purposes of our web diagram, we need to exchange $x$ and $y$ values, however--the function of our $x=y$ line originally. We, thus, solve for $x=\sqrt[4]{y}$, giving us $y=x^{4}$, which therefore serves as our reflective curve in the web diagram.

Reflective curves for other cases of sentences derivative or parasitic on self-referential sentences can be computed similarly. For pairs of sentences with algorithms $f^{\prime}$ and $f$ and inverses $f^{\prime-1}$ and $f^{-1}$ within the limits of our semantic interval $[0,1]$, given a value $b_{n}$ for the derivative sentence modeled by $f, b_{n+1}$ can be calculated by reflecting a $y$ value representing $b_{n}$ off the function given by:

$$
x=f^{\prime}\left(f^{-1}(y)\right) \quad \text { or } \quad y=f\left(f^{\prime-1}(x)\right)
$$

and recalculating the new $x$ value in terms of our function $f .^{12}$

To this point, we have concentrated on fuzzy relatives of the Liar. We should, however, also mention fuzzy relatives of the Truth-teller. Within classical logic, (7) proves troublesome in a manner different from but related to the troubles of the Liar:

This sentence is true.

Unlike the Liar, the difficulty with (7) is not that it cannot consistently be assigned a value of either $T$ or $F$. The difficulty with (7) is rather that it can consistently be assigned either value, and there seems no basis on which to prefer one over the other.

Within a fuzzy logic, using the algorithm

$$
x_{n+1}=x_{n}
$$

the Truth-teller can still consistently be assigned any value--in the fuzzy context, any numerical value in the $[0,1]$ interval.

${ }^{12}$ I leave to another context generalization to more complicated cases and to deeper chains, including sentences about the semantic value of sentences about the semantic value of $\cdots$ self-referential sentences. 


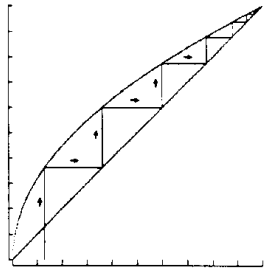

The Modest Truth-teller
for seed 13

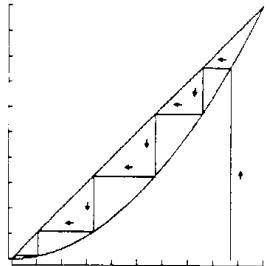

The Emphatic Truth-teller
for seed .87
Fig. 15 .

Consider also the Modest Truth-teller and the Emphatic Truth-teller:

This sentence is fairly true

This sentence is very true

with corresponding algorithms:

$$
\begin{aligned}
& x_{n+1}=\sqrt{x_{n}} \\
& x_{n+1}=x_{n}^{2} .
\end{aligned}
$$

Both Truth-tellers, like their classical predecessor, have fixed points at 0 and 1 . For fuzzy values in between, however, they vary dramatically and symmetrically. The self-reference of the Modest Truth-teller drives every intermediate value up to 1 . The Emphatic Truth-teller, on the other hand, drives every intermediate value down to 0 (Fig. 15).

The Truth-tellers, like the Modest Liar, thus in some sense force fuzziness to precision through the iteration of semantic self-reference. In the case of the Truth-tellers, as in the case of the Emphatic Liar, however, that inexorable self precision is the more remarkable since the values one is driven to in each case are classical values. In the case of the Truth-tellers, moreover, one is driven to stable classical values.

One lesson of sentences such as the Modest Truth-teller and the Emphatic Truth-teller is that dynamical semantics may introduce the need for logical categories beyond the traditional "tautology" and "contradiction." A classical tautology is one which (instantly, as it were) takes a value of " 1 " for any value assigned its components. A dynamic tautology, we might propose, is one which converges through iteration on a value of "l" for any initial value. The Modest Truth-teller might, thus, be proposed as a dynamic tautology; the Emphatic Truth-teller as a dynamic contradiction. ${ }^{13}$

From this first sampling of examples, it is clear that fuzzy self-reference opens up a realm of dynamical semantics far richer than anything dreamt of within classical logic. It is also clear that there are some surprises in the transition from classical to fuzzy logic. The Fuzzy Liar, for example, is a quite direct fuzzification of the standard Liar. Nonetheless, it

\footnotetext{
${ }^{13}$ The very different behavior of the Modest Truth-teller and the Emphatic Truth-teller is inevitable, given a modeling of "very" and "fairly" by squares and square roots-or, for that matter, by cubes and cube roots or the like. It has been suggested, however, that this is itself a mark against such a modeling: are "very" and "fairly" so different that "this is fairly true" should converge on pure truth and "this is very true" should converge on pure falsity?
}

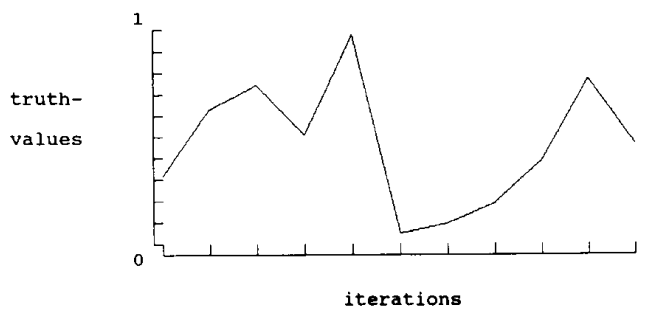

Fig. 16.

is not the Fuzzy Liar but the quite different Emphatic Liar that converges on the familiar oscillation between 0 and 1 .

It is also the case that fuzzy self-reference is capable of giving us full semantic chaos in the strict mathematical sense.

\section{FUZZY CHAOS}

Consider the following sentence, which $I$ will call the Chaotic Liar [8]:

This sentence is true if and only if it is false.

Using the Lukasiewicz biconditional and modeling selfreference in terms of iterated algorithms, semantic values for the Chaotic Liar will be given by:

$$
x_{n+1}=1-\operatorname{abs}\left(\left(1-x_{n}\right)-x_{n}\right) .
$$

By expanding our language slightly, we can also express the Chaotic Liar in other ways. If " $p$ is as true as $q$ " or "the value of $p$ is the same as the value of $q$ " are treated fairly naturally as taking the value

$$
1-\operatorname{abs}(\underline{v}(p)-\underline{v}(q))
$$

for example, then the Chaotic Liar can alternatively be expressed as:

This sentence is as true as it is false.

or

The value of this sentence is the same as its negation.

A seed value of 0.314 for any of these gives us a series of values that begins as in Fig. 16.

The semantical dynamics of the Chaotic Liar is perhaps best exhibited by its web diagram, however, here shown in four progressive stages of development ${ }^{14}$ (Fig. 17).

\footnotetext{
${ }^{14}$ One peculiarity of this function is that standard rounding off within the binary arithmetic of computers disguises its chaoticity: although it is probably chaotic on the $[0,1]$ interval, it does not show up as such on the computer screen. In order to graph something closer to the function's true behavior, it is thus standard to "cancel out" the effect of rounding off by introducing a small element of randomness, and that has been done for the illustrations here. On this point, I am obliged to J. Milnor for discussion.
} 

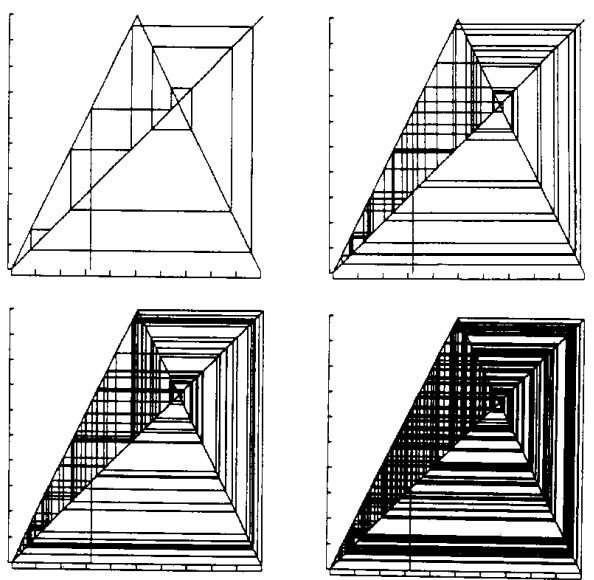

Fig. 17 .

The dynamical semantics of the Chaotic Liar qualifies as chaotic in the precise mathematical sense of [36]. ${ }^{15}$

What this amounts to is a range of surprising semantic features.

1) The Fuzzy Liar, as we have seen, has a simple period of 2 for almost all values. For sentences such as the Chaotic Liar, on the other hand, any repeating period we might care to choose, however high, will be generated by some initial value. For such sentences, there is no upper limit to semantic periodicity.

2) It is also the case that there will be numerical truth values for such sentences which eventually move from any arbitrarily small semantic region to any other. There is, thus, no range of degrees of truth, however small, such that values within that range assigned to the Chaotic Liar will safely stay there on iteration. For any such region, some semantic values will eventually escape to any other semantic region we might name.

3 ) Finally, no matter what numerical truth value $x$ we might start off with as an estimate for such sentences, there will be numerical truth values arbitrarily close to our initial value which, upon iterative recalculation through our sentences, eventually move as far from corresponding iterations of $x$ as we might choose to specify. There is, thus, no initial range "close enough" to a starting estimate $x$ that differences

${ }^{15}$ Given a set $J, f: J \rightarrow J$ is chaotic on $J$ if: a) $f$ shows sensitive dependence on initial conditions, $f$ b) $f$ is topologically transitive, and c) the set of periodicpoints is dense in $J$.

Here, let us use the notation $F^{n}(x)$ to stand for the composition or iteration of the function $f(x) n$ times, i.e.,

$$
f^{n_{2}}(x)=\underbrace{\cdots f(f(f(x))) \cdots} n \text { times }
$$

a) $f: J \rightarrow J$ shows sensitive dependence on initial conditions if there exist points arbitrarily close to any $x \in J$ which eventually separate from $x$ by any chosen distance $\delta$ or more under iteration of $f$, i.e., there exist $\delta>0$ such that, for any $x \in J$ and any neighborhood $N$ of $x$, there exist $y \in N$ and $n \geq 0$ such that abs $\left(f^{n}(x)-f^{n}(y)\right)>\delta$.

b) $f: J \rightarrow J$ is topologically transitive if it has points which eventually move under iteration from one arbitrarily small neighborhood to any other, i.e., for any pair of open sets $L . V \subseteq J$ there exists some $n>0$ such that $f^{n}(U) \cap V$ is nonempty.

c) The set of periodic points of $J, \operatorname{PER}(J)$, is the set of all $r \in J$ such that $f^{n}(x)=x$ for some natural number $n$, i.e., $\operatorname{PER}(J)=\{x \in J$ : $\left.\exists n f^{n}(x)=x\right\} . \operatorname{PER}(J)$ is dense in $J$ if $\operatorname{PER}(J)$, together with all its limit points, is equal to $J$.

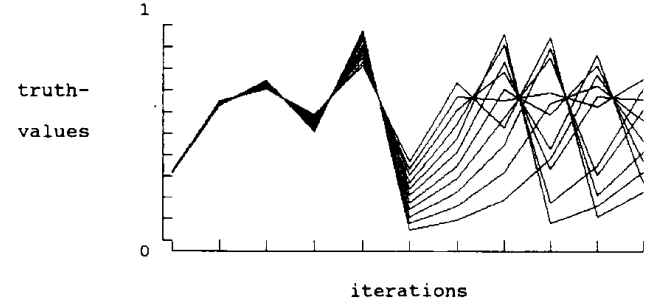

Fig. 18.

within that range will not make a significant difference. Within any distance from $x$, however small, is another value iterations of which will eventually diverge from iterations of $x$ enormously--as enormously, within the limits of our semantic range, as we might care to specify.

Although somewhat stronger and weaker characterizations of chaos appear in the literature, the central element in all versions is this last feature, known as sensitive dependence on initial conditions. The graph in Fig. 18 shows the basic idea of sensitive dependence for the Chaotic Liar. Here, time series graphs are superimposed for seed values tarting with 0.314 and increasing by 0.001 .

The basic algorithm for the Chaotic Liar is, in fact, a very simple and paradigmatically chaotic function, known as a tent map because of the shape of its graph and more familiar in the mathematical guise $x_{n+1}=1-\operatorname{abs}\left(2 x_{n}-1\right)$ or

$$
x_{n+1}= \begin{cases}2 x_{n} & \text { for } 0 \leq x<0.5 \\ 2\left(1-x_{n}\right) & \text { for } 0.5 \leq x \leq 1\end{cases}
$$

(see [37], $171 \mathrm{ff}$.]). The semantic role of this function within self-referential fuzzy logic comes as something of a surprise, however; the function is, for example, relegated to a mere class of "mathematical curiosities" in [38]. ${ }^{16}$

Here, let me also offer a second simple sentence which shows chaotic behavior within fuzzy logic--a sentence I will call the Fuzzy Logistic:

It is very false that this sentence is true if it is false.

Here, semantic values will be given by:

$$
x_{n+1}=\left(1-\left(1-\operatorname{abs}\left(\left(1-x_{n}\right)-x_{n}\right)\right)\right)^{2} .
$$

Note that the algorithm for the Chaotic Liar is embedded within that of the Fuzzy Logistic. We can, in fact, obtain the Fuzzy Logistic from the Chaotic Liar simply by adding the prefix "It is very false that ...".

Here, as before, there are of course alternative phrasings. If we take "differs in value from" as the negation of "is as true as," the Fuzzy Logistic can be phrased as:

It is very true that this sentence differs in value from its negation.

or

The value of this sentence is very different from that of its negation.

\footnotetext{
${ }^{16}$ The similar role of that algorithm in a Rescher multivalued logic appears in [8], [9].
} 

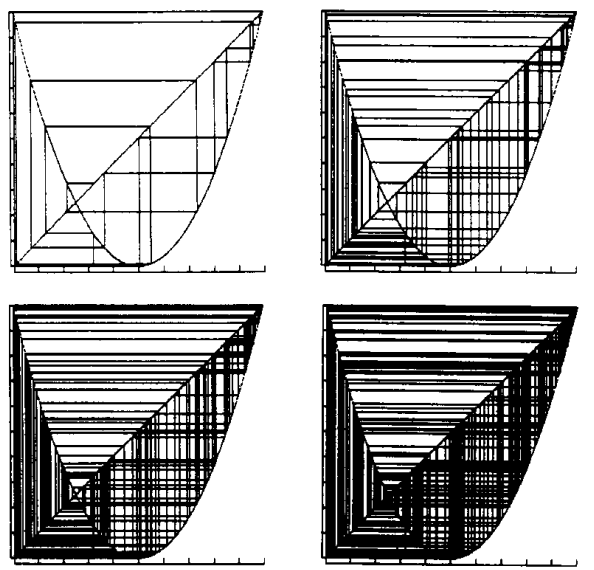

Fig. 19.

For values in the $[0,1]$ interval, our algorithm amounts in each case to:

$$
\begin{aligned}
x_{n+1} & =\left(\left(1-x_{n}\right)-x_{n}\right)^{2} \\
& =\left(1-2 x_{n}\right)^{2} \\
& =1-4 x_{n}\left(1-x_{n}\right) .
\end{aligned}
$$

This, it turns out, is an inverted form of the logistic or quadratic equation, perhaps the most familiar and thoroughly studied sample of chaos. ${ }^{17}$

For an initial value of 0.314 , the web diagram of the Fuzzy Logistic develops as in Fig. 19.

Chaos can be expected to appear within other fuzzy logics by way of other self-referential sentences. Though we have here confined ourselves in general to the simple Baldwin fuzzy logic outlined in Section II, it is perhaps worth noting a route by which chaos will appear within any fuzzy logic with the standard Lukasiewicz base, regardless of the fuzzy set it introduces for "true." Within Zadeh fuzzy logic or any other based on $L_{\aleph 1}$, consider the prospect of a sentence $p$ which amounts to a biconditional between itself and its negation:

$$
p=(p \leftrightarrow \sim p) .
$$

Given simply the basic Lukasiewicz biconditional, the algorithm for such a sentence will be:

$$
x_{n+1}=1-\operatorname{abs}\left(\left(1-x_{n}\right)-x_{n}\right)
$$

\footnotetext{
${ }^{17}$ An additional negation would give us the Logistic without inversion It is not very false that this sentence is true if it is false

The value of this sentence is not very different from that of its negation I am obliged to N. Hellerstein for his seminal work on the Fuzzy Logistic, communicated in private correspondence.
}

and any such sentence will, thus, amount to the Chaotic Liar. $^{18}$

\section{FuZZY SELF REFERENCE IN Two Dimensions}

Beyond the traditional Liar lies an infinite series of Liarcycles, the simplest of which is perhaps the Dualist. In medieval form, it appears as an exchange between Socrates and Plato:

Socrates: What Plato is about to say is true.

Plato: Socrates speaks falsely.

More simply, we have two sentences each of which is about the truth value of the other:

$$
\begin{aligned}
& X: Y \text { is true } \\
& Y: X \text { is false. }
\end{aligned}
$$

With the tools of our basic fuzzy logic, we can also introduce fuzzy variations on the Dualist. Consider, for example,

$$
\begin{aligned}
& X: X \leftrightarrow Y \\
& Y: Y \leftrightarrow \text { it is very false that } X
\end{aligned}
$$

or, equivalently,

$$
\begin{aligned}
& X: X \text { is true if and only if } Y \text { is } \\
& Y: Y \text { is true if and only if } X \text { is very false. }
\end{aligned}
$$

In previous sections, we have concentrated on single sentences which force a series of revised values. Here, the situation is somewhat more complex: we have a pair of sentences which, for any initial seed values $\left(x_{0}, y_{0}\right)$, forces a series of pairs of revised values. For $X$ and $Y$, revised values can be calculated in terms of the following algorithms:

$$
\begin{aligned}
& x_{n+1}=1-\operatorname{abs}\left(x_{n}-y_{n}\right) \\
& y_{n+1}=1-\operatorname{abs}\left(y_{n}-\left(1-x_{n}\right)^{2}\right) .
\end{aligned}
$$

If we start with seed values of 0.25 and 0.25 , for example, we are forced to the following series of revised values: ${ }^{19}$

$$
\begin{gathered}
(1,0.6875),(0.6875,0.3125),(0.625,0.7852), \\
(0.8398,0.3555),(0.5156,0.6702) \cdots
\end{gathered}
$$

\footnotetext{
${ }^{18}$ For Zadeh fuzzy logic in particular, St. Denis has suggested the following
} chaotic sentence:

It is not the case thąt this sentence is fairly not true, or it is not the case that the negation of this sentence is fairly not true.

Here, our algorithm is:

$$
\left.\left.x_{n+1}-\max \left[1-\sqrt{\left(1-Z\left(x_{n}\right)\right.}\right), 1-\sqrt{\left(1-Z\left(1-x_{n}\right)\right.}\right)\right]
$$

where $Z\left(x_{n}\right)$ indicates the degree of membership in Zadeh's fuzzy set "true" (using 0.5 as $\alpha$ ) of a sentence with numerical truth value $x_{n}$. In a web diagram, the $\$$ t. Denis sentence appears as a more slender variation on the inverse Logistic.

${ }^{19}$ Here again, numbers are rounded off for presentational simplicity. 


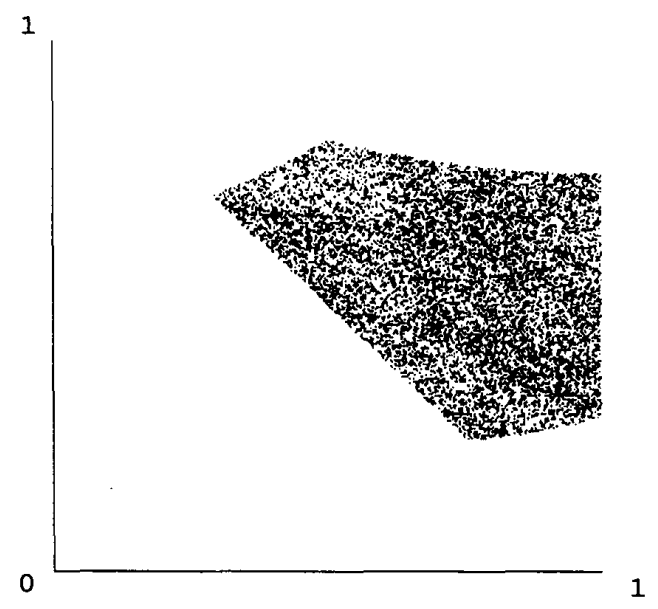

Fig. 20 .

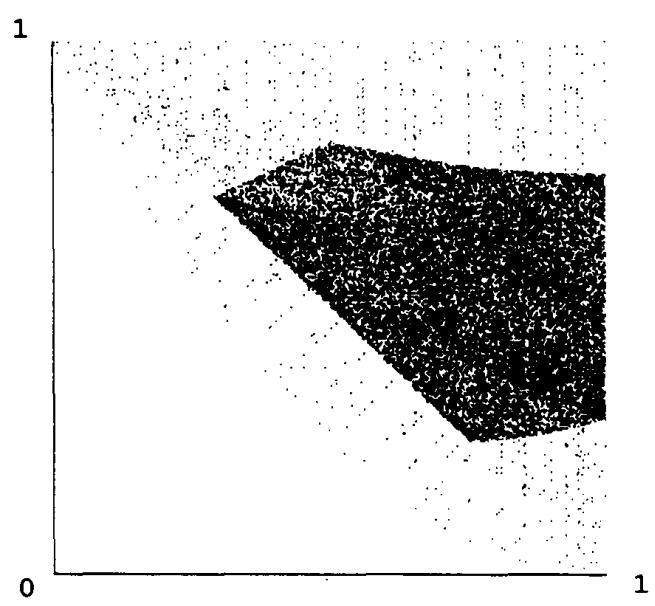

Fig. 21 .

If we plot these pairs as Cartesian coordinates, the pentagonal attractor of Fig. 20 appears.

The persistence of such an attractor, for various seed values, is clear from an overlay diagram for seed values $(x, y)$ where $x$ and $y$ range from 0 to 1 in intervals of 0.5 (Fig. 21). Throughout the $[0,1]$ interval, values are attracted to and trapped within the same clearly defined region.

Consider, also, a second fuzzy Dualist variation:

$$
\begin{aligned}
& X: \text { It is very false that } X \leftrightarrow Y \\
& Y: \text { It is fairly false that } Y \leftrightarrow \sim X
\end{aligned}
$$

or, more colloquially,

$X:$ It is very false that $X$ is true if and only if $Y$ is.

$Y:$ It is fairly false that $Y$ is true if and only if $X$ is false.

Here, successive values can be calculated using the following algorithms:

$$
\begin{aligned}
& x_{n+1}=\left(1-\left(1-\operatorname{abs}\left(x_{n}-y_{n}\right)\right)\right)^{2} \\
& y_{n+1}=\sqrt{1-\left(1-\operatorname{abs}\left(y_{n}-\left(1-x_{n}\right)\right)\right)}
\end{aligned}
$$

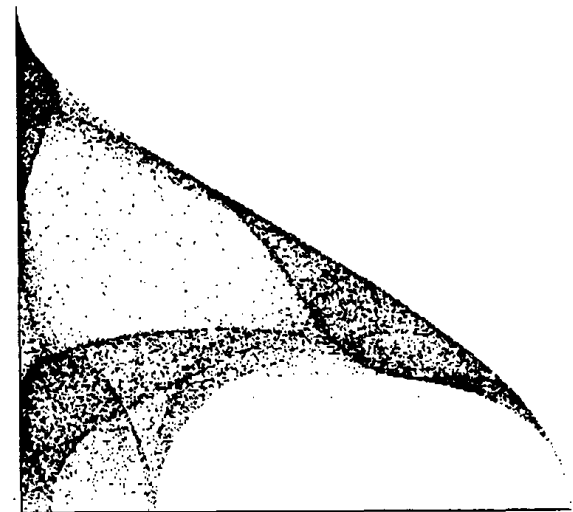

Fig. 22 .

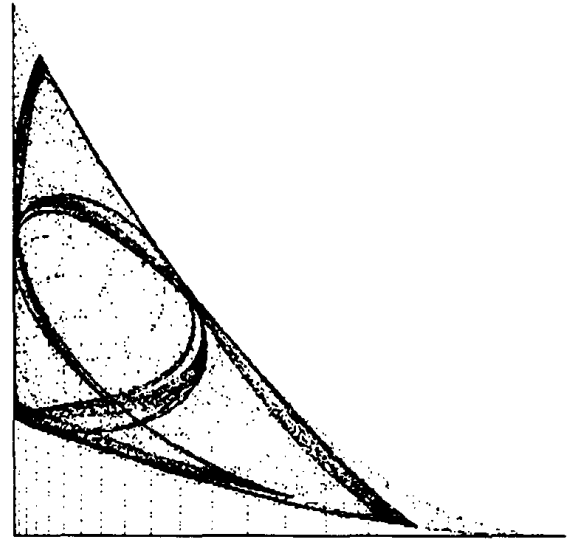

Fig. 23.

or, more simply,

$$
\begin{aligned}
x_{n+1} & =\left(x_{n}-y_{n}\right)^{2} \\
y_{n+1} & =\sqrt{\operatorname{abs}\left(y_{n}-\left(1-x_{n}\right)\right)} .
\end{aligned}
$$

Our attractor in this case is shown in an overlay diagram in Fig. 22.

Here, let me finally offer one further fuzzy Dualist:

$$
\begin{aligned}
& X: \text { It is very false that } X \leftrightarrow Y \\
& Y: \text { It is very false that } X \text { is false if and only if } \\
& Y \text { is very true }
\end{aligned}
$$

with successive values

$$
\begin{aligned}
x_{n+1} & =\left(x_{n}-y_{n}\right)^{2} \\
y_{n+1} & =\left(\left(1-x_{n}\right)-y_{n}^{2}\right)^{2} .
\end{aligned}
$$

The attractor for this final variation, once again in overlay form, appears in Fig. 23.

In our calculation of revised values for the Fuzzy Dualists, it should be noted we have assumed a simultaneous calculation of numerical truth values for sentences $X$ and $Y$ in each case. Given a pair of seed values $(x, y)$, in other words, we have calculated a new value for $X$ in terms of those values and 
have simultaneously calculated a new value for $Y$ in terms of those same values.

Evaluation of the sentences of a Fuzzy Dualist pair might also be thought of sequentially. It might be argued, for example, that in at least some contexts a more natural way to approach such a pair of sentences would be to begin with seed values $(x, y)$, to calculate the value of $X$ in terms of those seed values, but then to calculate the value of $Y$ using the newer or most recent value computed for $X$.

This second pattern of reasoning, with regard to the same pairs of sentences, can be represented with a slight change in our algorithms: In each case, $x_{n}$ is replaced in the second algorithm with $x_{n+1}$.

Our algorithms for the first variation on the Dualist, for example,

$$
\begin{aligned}
& X: X \leftrightarrow Y \\
& Y: Y \leftrightarrow \text { it is very false that } X
\end{aligned}
$$

will now be

$$
\begin{aligned}
& x_{n+1}=1-\operatorname{abs}\left(x_{n}-y_{n}\right) \\
& y_{n+1}=1-\operatorname{abs}\left(y_{n}-\left(1-x_{n+1}\right)^{2}\right) .
\end{aligned}
$$

Using this alternative form of calculation, the same pair of sentences give us the attractor shown in Fig. 24. A similar change from a simultaneous to sequential pattern of reasoning, in the case of our other fuzzy Dualist variations gives us the following attractors. As shown in Fig. 25:

$$
\begin{aligned}
& X: \text { It is very false that } X \leftrightarrow Y \\
& Y: \text { It is fairly false that } Y \leftrightarrow \sim X \\
& x_{n+1}=(x-y)^{2} \\
& y_{n+1}=\sqrt{\operatorname{abs}\left(y_{n}-\left(1-x_{n+1}\right)\right)} .
\end{aligned}
$$

As shown in Fig. 26.

$$
X: \text { It is very false that } X \leftrightarrow Y
$$

$Y:$ It is very false that $X$ is false if and only if

$Y$ is very true

$$
\begin{aligned}
x_{n+1} & =\left(x_{n}-y_{n}\right)^{2} \\
y_{n+1} & =\left(\left(1-x_{n+1}\right)-y_{n}^{2}\right)^{2}
\end{aligned}
$$

Although these figures graphically illustrate the difference between simultaneous (or parallel) and sequential updating in calculating the fuzzy Dualist, it must be confessed that their interpretation remains much less clear. Simultaneous updating might be said to be more appropriate to a God's-eye view of the informational dynamics of the fuzzy Dualist, in which all information is received and processed simultaneously at each step. Sequential updating, on the other hand, might be said to be more appropriate to beings capable of processing only the information of a single sentence at a time. In more realistic applications to repeated sequences of mutually referential sentences, the difference might be appropriate to contexts in which two sources of information provide information about each other, and in which the second source of information

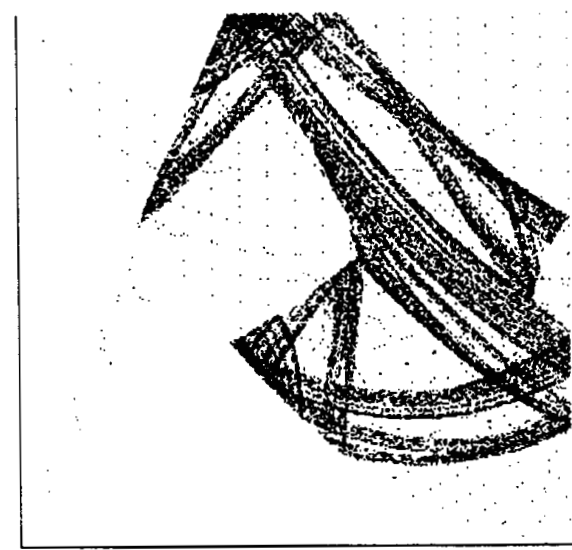

Fig. 24.

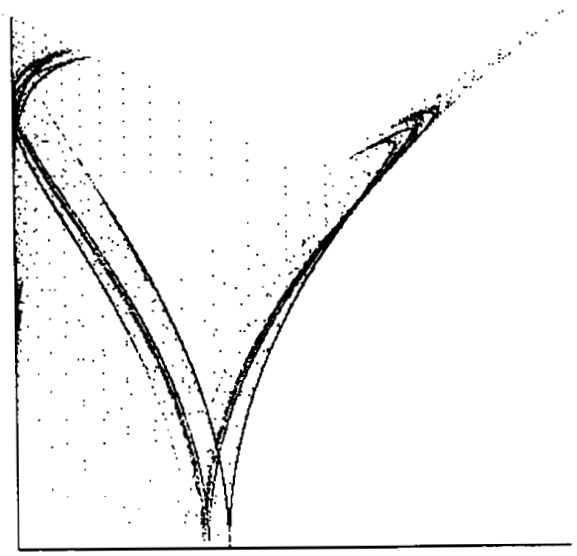

Fig. 25 .

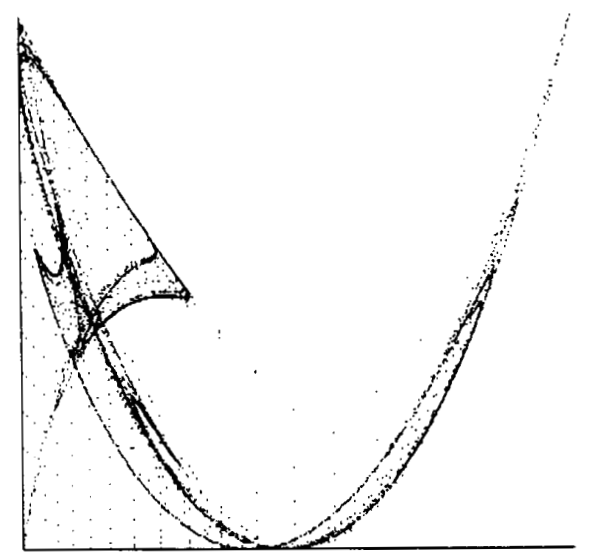

Fig. 26.

is or is not immediately aware of reports coming from the first source. All of this remains at the level of speculation, however: here, as elsewhere, the graphic display of semantic characteristics is as yet clearer than are matters of their interpretation or application. 
1

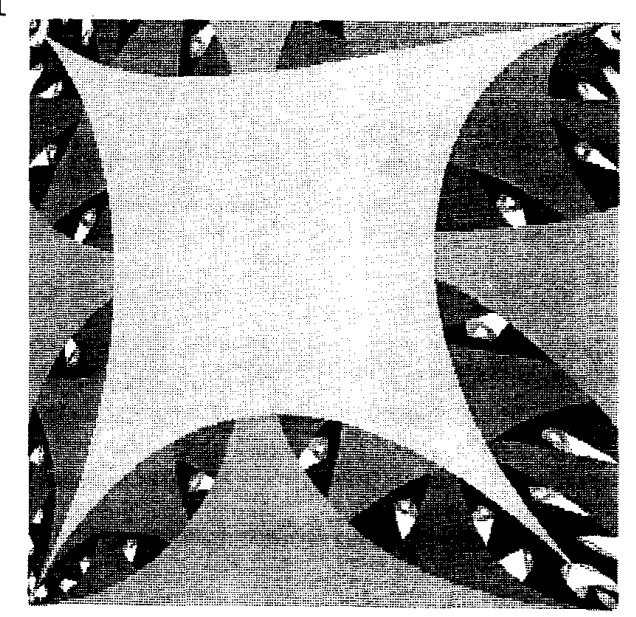

0

Fig. 27.

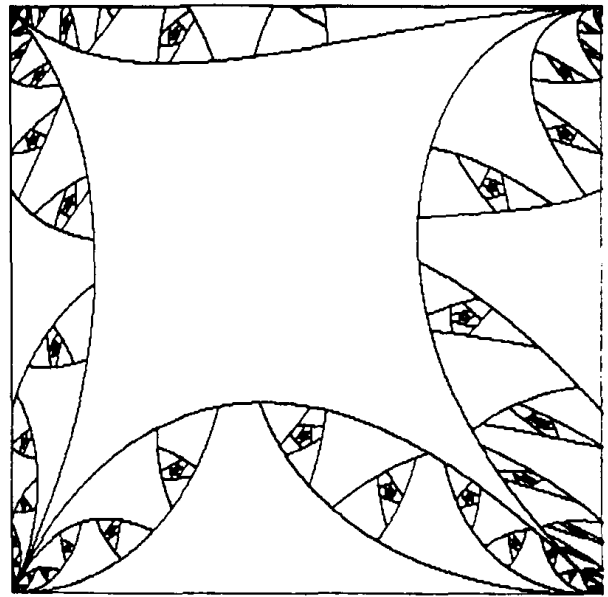

Fig. 28.

Certain aspects of the fuzzy dynamics of Dualist variations can also be graphed using what are known as escape-time diagrams. For each pair of points $(x, y)$ on the Cartesian plane, we can graph in terms of color the number of iterations required for the series of values, beginning with seed values $(x, y)$, to reach a certain threshold. Here, one threshold we might choose is a certain distance from the origin, with the origin itself representing $(0,0)$ or "double falsity" for our two sentences. Within a particular fuzzy Dualist, a pair of seed values (say $0.1,0.5$ ) may give us a series of values which first escapes from a distance of 1 from the origin in two iterations, for example, in three, in four, or in more. If that series escapes in two iterations, we might color the initial point $(0.1,0.5)$ blue; if three, we might color it green, and so forth. Another point (say $0.2,0.4$ ) may give us a series which escapes our chosen threshold in a different number of iterations, and will correspondingly be given a different color. (The general idea of escape-time diagrams should be familiar

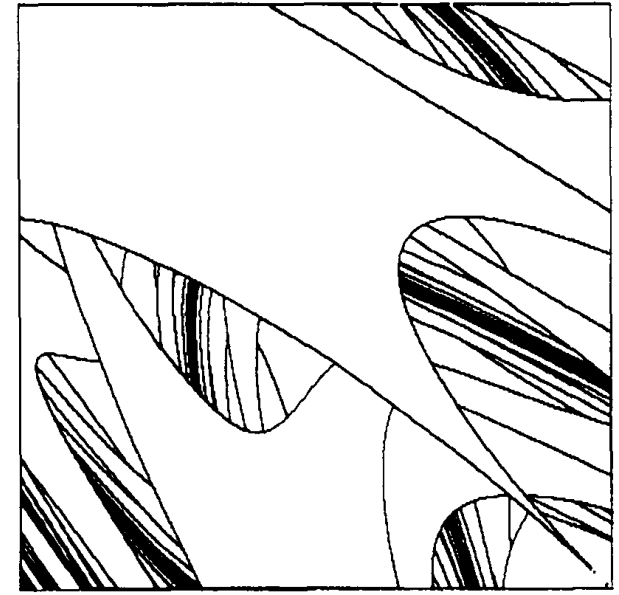

Fig. 29
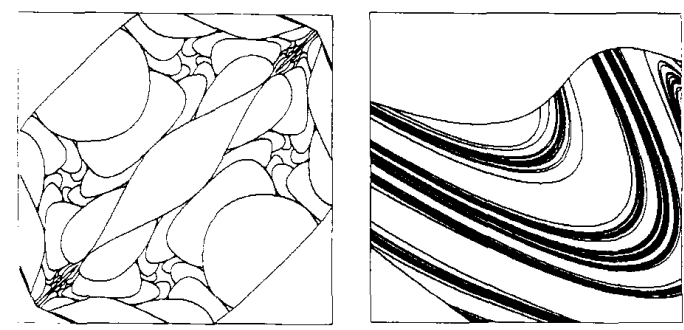

Fig. 30.

from standard graphing of the Julia and Mandelbrot sets.)

Fig. 27 shows an escape-time diagram of this type for the first fuzzy Dualist.

Given present printing limitations, however, the fractal character of such a graph is perhaps clearer if we emphasize merely the interfaces of different colored areas: points at which the number of iterations required to pass the chosen threshold changes. Our escape-time diagram now appears as a tracery (Fig. 28).

Consider, in contrast, an escape-time diagram for a sequential pattern of reasoning with regard to our first Fuzzy Dualist (Fig. 29).

Fig. 30 shows escape-time diagrams for simultaneous and sequential calculations (left and right, respectively) of the second Dualist variation offered.

$$
\begin{aligned}
& X: \text { It is very false that } X \leftrightarrow Y . \\
& Y: \text { It is fairly false that } Y \leftrightarrow \sim X .
\end{aligned}
$$

In this case, an escape threshold of 0.8 is used.

In all of the escape-time diagrams considered to this point, we have confined our values $(x, y)$ to the unit interval, reflecting the fact that the semantics of our fuzzy logic limits numerical truth values to the $[0,1]$ interval. In some cases, however, it is easy to see that the characteristics of points within the $[0,1]$ interval are merely part of a larger pattern. 


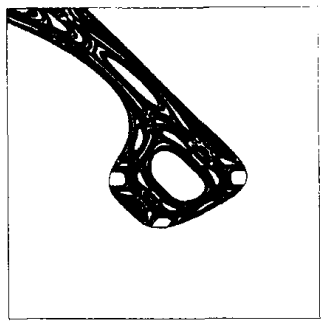

-inultaneous

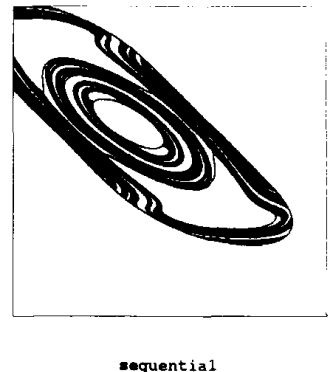

Fig. 31.
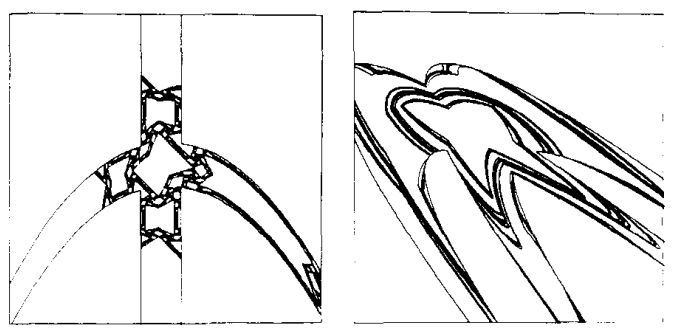

Fig. 32.

Fig. 31 shows our third Fuzzy Dualist variation for values between -1.4 and 2.4 and with a threshold of $0.85:^{20}$

$$
\begin{aligned}
& X: \text { It is very false that } X \leftrightarrow Y \\
& Y: \text { It is very false that } X \text { is false if and only if } \\
& Y \text { is very true }
\end{aligned}
$$

Other escape-time diagrams are possible in terms of other parameters. In all those mentioned, we noted the threshold used is a particular distance of a pair $(x, y)$ from the origin. We can also plot escape-time diagrams using other thresholds as well. Fig. 32 shows an escape-time diagram in which what we measure is the number of iterations required before a series starting from a pair of seed values $(x, y)$ reaches a value $\left(x^{\prime}, y^{\prime}\right)$ such that $x$ and $y$ are separated by a distance of at least 0.5 .

In many of these images, a deep fractal character--self affinity at descending scales--is clearly evident. This is a familiar companion to chaos within dynamical systems theory. What its appearance indicates, however, is the presence of fractal organization in the dynamical semantics of self-referential sentences within a fuzzy logic. It is tempting to speculate that different varieties of self-reference, direct or indirect, can themselves be thought of as abstractly fractal in some intuitive sense: self-referential sentences or sets of sentences semantically contain themselves, or images of themselves, in much the way that fractals seem to contain themselves on different scales. It might be proposed that what images such as those in Fig. 32 really do is give more explicit visual expression to the inherently fractal semantics of different patterns of self-reference. This remains speculation, however. Here, as elsewhere, it proves easier to graph certain semantic characteristics than to fully understand them.

${ }^{20}$ The range of Fuzzy Dualist variations is so immense as to be intimidating For a few other small samples, the reader is referred [8], [9], [33].

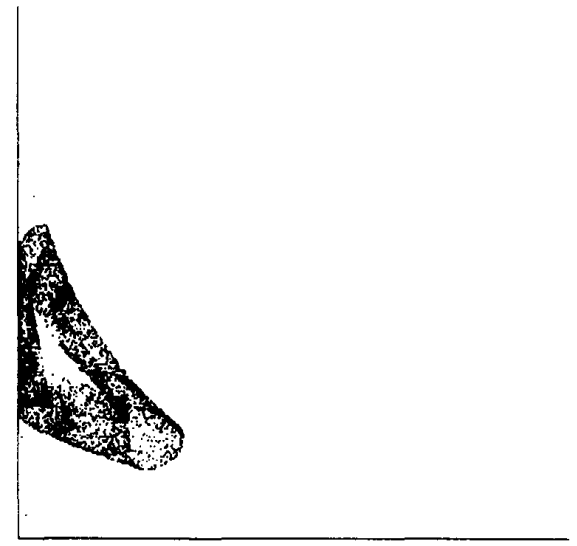

Fig. 33 .

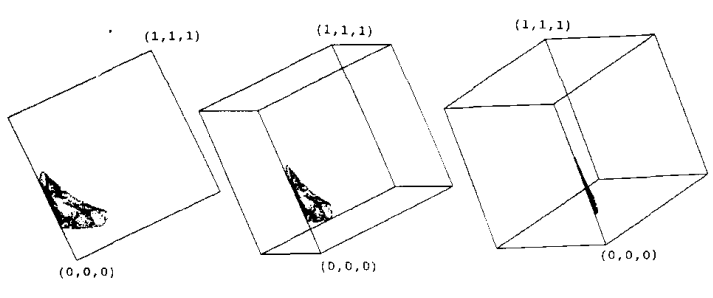

Fig. 34 .

\section{FuZZY TRIPLISTS Modeled IN Three Dimensions}

Beyond the Dualist lie Triplist variations, in which three mutually referential sentences speak of each others' truth values. In the case of Triplists, attractors must be graphed as three-dimensional rather than two-dimensional objects, and the correlates to two-dimensional escape-time diagrams will be three-dimensional escape-time solids. Here again, I simply offer some examples.

Consider, to begin with, the following set of sentences (a colon is used to avoid ambiguity):

$$
\begin{aligned}
& X: \text { It is very false that: } X \leftrightarrow \sim(Y \leftrightarrow Z) \\
& Y: \text { It is very false that: } Y \leftrightarrow \sim Z \\
& Z: \text { It is very false that: } Z \leftrightarrow \sim(X \leftrightarrow Y) .
\end{aligned}
$$

In the fuzzy Dualists, our sentences forced us through a series of revisions for initial seed values $(x, y)$ for sentences $X$ and $Y$. In the case of this fuzzy Triplist, our sentences will force us through a similar series of revisions for seed values $(x, y, z)$. For these sentences, these revised values can be calculated in terms of the following algorithms:

$$
\begin{aligned}
& x_{n+1}=\left(\operatorname{abs}\left(y_{n}-z_{n}\right)-x_{n}\right)^{2} \\
& y_{n+1}=\left(\left(1-z_{n}\right)-y_{n}\right)^{2} \\
& z_{n+1}=\left(\operatorname{abs}\left(x_{n}-y_{n}\right)-z_{n}\right)^{2} .
\end{aligned}
$$

If we plot revised values for these sentences starting with seed values of $(0.23,0.34,0.45)$, the attractor of Fig. 33 appears.

In Fig. 33, the attractor is shown in two dimensions ("fullface," as it were, from the $z$ axis). In Fig. 34, in contrast, it is rotated in three dimensions. Despite its apparent depth, it is clear that the attractor for this first fuzzy Triplist is still confined to a plane. 


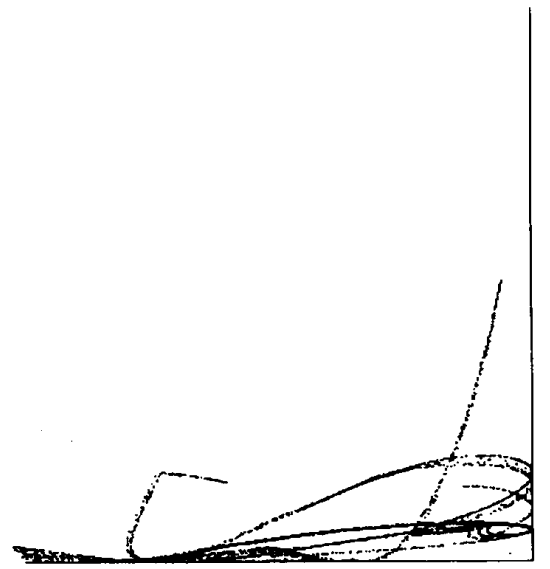

Fig. 35.

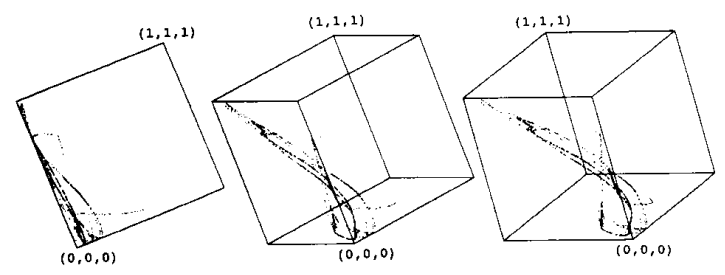

Fig. 36.

Here, as before, we can also compute revised values for oursentences by considering them sequentially rather than simultaneously, with the following changes in our algorithms:

$$
\begin{aligned}
& x_{n+1}=\left(\operatorname{abs}\left(y_{n}-z_{n}\right)-x_{n}\right)^{2} \\
& y_{n+1}=\left(\left(1-z_{n}\right)-y_{n}\right)^{2} \\
& \left.z_{n+1}=\operatorname{abs}\left(x_{n+1}-y_{n+1}\right)-z_{n}\right)^{2} .
\end{aligned}
$$

With a sequential calculation, the looping attractor of Figs. 35 and 36 appears.

Consider a second fuzzy Triplist variation:

$$
\begin{aligned}
& X: \sim(X \leftrightarrow \text { it is very true that } Y \leftrightarrow Z) \\
& Y: \sim(Y \leftrightarrow \text { it is very true that } X \leftrightarrow Z) \\
& Z: \sim(Z \leftrightarrow \text { it is very true that } X \leftrightarrow Y)
\end{aligned}
$$

with the following algorithms for revised values:

$$
\begin{aligned}
& x_{n+1}=\operatorname{abs}\left(\left(1-\operatorname{abs}\left(y_{n}-z_{n}\right)\right)^{2}-x_{n}\right) \\
& y_{n+1}=\operatorname{abs}\left(\left(1-\operatorname{abs}\left(x_{n}-z_{n}\right)\right)^{2}-y_{n}\right) \\
& z_{n+1}=\operatorname{abs}\left(\left(1-\operatorname{abs}\left(x_{n}-y_{n}\right)\right)^{2}-z_{n}\right) .
\end{aligned}
$$

Using the same seed values as before, this second fuzzy Triplist gives us the attractor of Figs. 37 and 38. A sequential computation, in contrast, gives us Figs. 39 and 40.

For fuzzy Triplists, the analog to two-dimensional escapetime diagrams will be three-dimensional escape-time solids. We can once again color points in terms of how many iterations

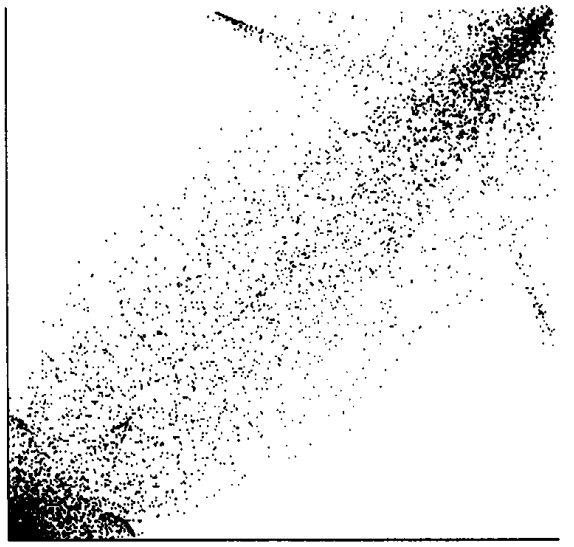

Fig. 37.

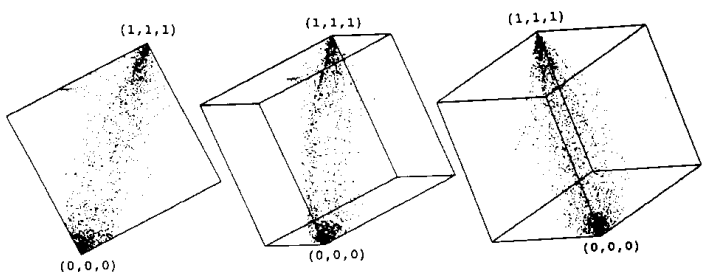

Fig. 38.

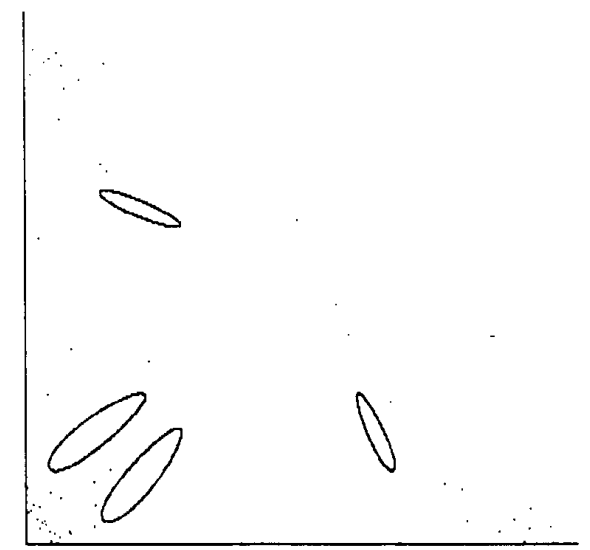

Fig. 39.

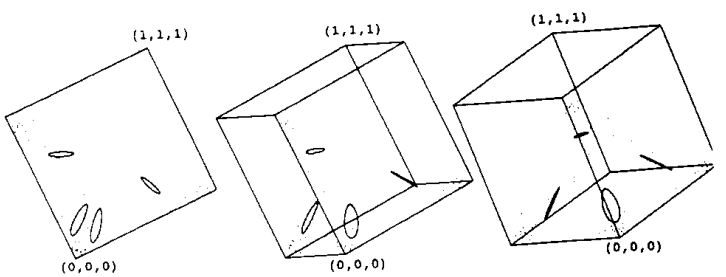

Fig. 40.

are required for a series of revised values starting from that point to reach a certain threshold. In the case of Triplist variations, however, we will be coloring points $(x, y, z)$ in a three-dimensional space. 

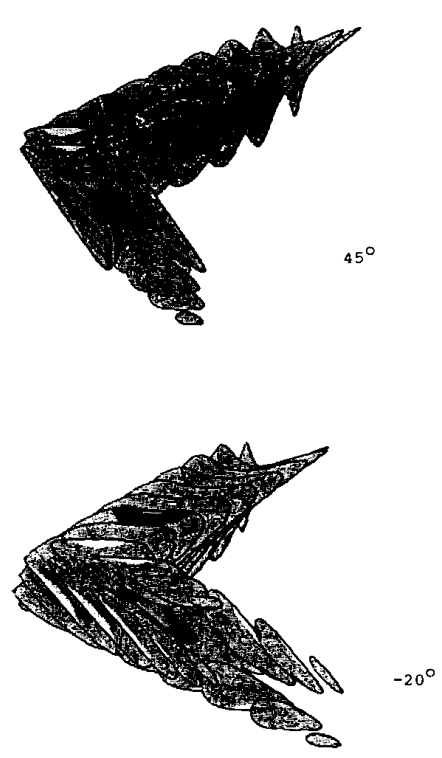

In Figs. 41 and 42, we use sequential calculation for the first Triplist variation and simultaneous calculation for the second, with a chosen threshold in each case of $\sqrt{x^{2}+y^{2}+z^{2}}=1$. Both escape-time solids are shown from two angles, in a space extending roughly from -2.5 to +5 for each of our three values:

$$
\begin{aligned}
& X: \text { It is very false that: } X \leftrightarrow \sim(Y \leftrightarrow Z) \\
& Y: \text { It is very false that } Y \leftrightarrow \sim Z \\
& Z: \text { It is very false that: } Z \leftrightarrow \sim(X \leftrightarrow Y)
\end{aligned}
$$

$$
\begin{aligned}
& X: \sim(X \leftrightarrow \text { it is very true that } Y \leftrightarrow Z) \\
& Y: \sim(Y \leftrightarrow \text { it is very true that } X \leftrightarrow Z) \\
& Z: \sim(Z \leftrightarrow \text { it is very true that } X \leftrightarrow Y) .
\end{aligned}
$$

There is no upper limit to the size of sets of mutually self-referential sentences that might be considered, of course, nor any upper limit to the number of dimensions appropriate for modeling their semantical dynamics. Beyond the threedimensional semantic phenomena of Triplist variations lie the four-dimensional semantic phenomena of the Quadruplists, the five-dimensional semantic phenomena of Quintuplists, and so on.

\section{CONCLUSION}

Here, my attempt has been to introduce, for the most part by example, a range of dynamical phenomena which appear in the semantics of a simple fuzzy logic with the introduction of self-reference. Within such a logic appear sentences the dynamical semantics of which exhibit the behavior of fixed point attractors, fixed point repellers, and full chaos on the $[0$, 1] interval of semantic values. Mutually referential Dualist and Triplist pairs take the phenomena of chaos and fractals, once again in a semantic guise, into two and three dimensions.
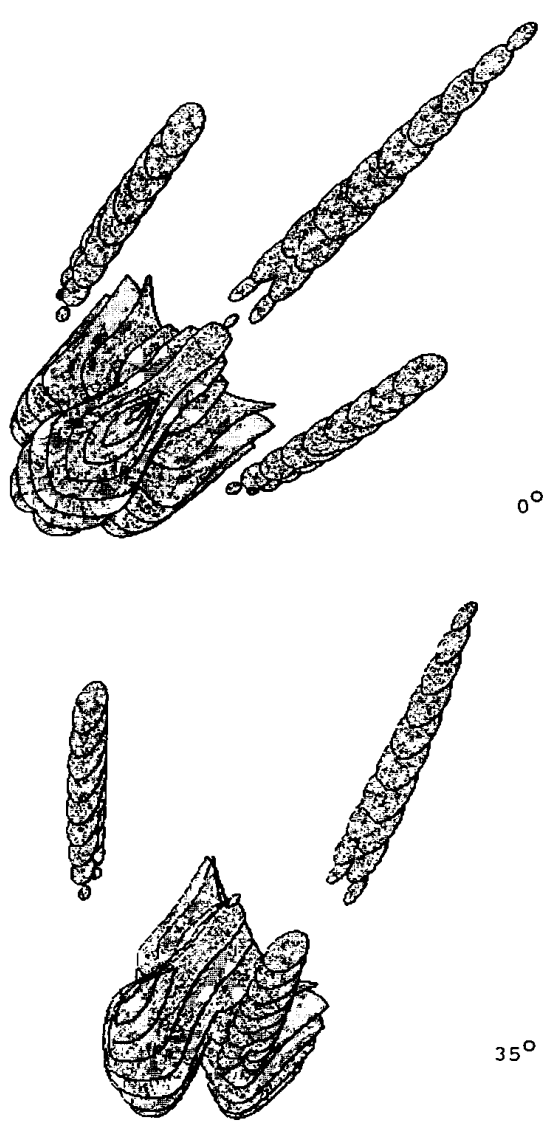

Fig. 42.

A great deal of further formal exploration, generalization, andapplication clearly remains to be done. ${ }^{21}$ Perhaps it is not out of place, however, to close with some admittedly philosophical speculations.

Logical systems have typically been introduced with certain semantical expectations, and one thing the introduction of semantical self-reference often does is to violate those initial expectations. Here, classical logic is a prime example: within such a logic, the expectation is that every proposition will be simply true or false. With the introduction of semantical self-reference, however, we are confronted with the classical Liar:

This sentence is false.

The dynamical semantics of such a sentence seems to be that of an oscillation, and the attempt to assign either of our supposedly exhaustive semantical categories results in simple contradiction. A similar story, relying on strengthened versions of the Liar, can be told for multivalued, infinite-valued, gapped, and antifoundational logics $[1, \mathrm{ch} .1]$. In each case,

${ }^{21}$ One metamathematical application is mentioned in the introduction: [8], [9] each contain a sketch of Gödel-like limitative results for chaos theory in the context of formal systems for real arithmetic, motivated by a close relative of the sentence that appears here as the Chaotic Liar. It is clear that one class of extensions would take these into the context of fuzzy logics. 
self-reference seems to violate initial semantical expectations by forcing us to recognize categories of semantical behavior not initially and intuitively provided for.

What the work above seems to show is that something similar also happens with the introduction of semantical selfreference within fuzzy logics. Fuzzy logic was, of course, constructed to incorporate an important range of intuitive phenomena and to facilitate a range of applications not provided for within more classical logics. One assumption which appears to have been carried over from its classical predecessors, however, was that semantic values, however fuzzy, could nonetheless be expected to be tolerably well behaved and manageably stable. Here again, the introduction of semantical self-reference seems to violate central semantical expectations in the context of fuzzy logic, self-reference seems to introduce a range of patterns of semantic instability as diverse and complex as the phenomena of chaos theory generally.

\section{ACKNOWLEDGMENT}

The work presented here is an expansion of collaborative work on infinite-valued logics and chaos which appears in Mar and Grim [8], Grim and Mar [9], and Grim, Mar, Neiger, and St. Denis [23]. The author is indebted to P. St. Denis for programming assistance and for repeatedly bringing my attention back to the Lukasiewicz biconditional. M. Neiger developed the programming required for three-dimensional escape-time solids in Section V. As always, the author is deeply indebted to G. Mar for fruitful discussion and for central good ideas. The author would also like to express thanks to several anonymous referees for detailed and helpful comments.

\section{REFERENCES}

[1] J. J. Buckley, "Fuzzy dynamical systems: I." in Proc. IFSA '91, Brussels, Belgium, pp. 16-20.

[2] G.-Y. Wang, J.-P. Ou, and P.-Z. Wang, "Dynamic fuzzy sets and fuzzy processes," in Proc. 3rd IFSA Cong., Seattle, WA, 1989, pp. 276-279.

[3] P. Diamond, "Chaos and fuzzy representations of dynamical systems," in Proc. Int. Symp. Fuzzy Syst., Iizuka, Japan, July 1992, pp. 51-58.

[4] P. Grim, The Incomplete Universe. Cambridge, MA: M.I.T. Press, 1991.

[5] H. Herzberger, "Notes on naive semantics," J. Phil. Log., vol. 11, pp. 61-102, 1982.

[6] A. Gupta, "Truth and paradox," J. Phil. Logic, vol. 11, pp. 1-60, 1982.

[7] A. Gupta, and N. Belnap, The Revision Theory of Truth. Cambridge, MA: M.I.T. Press, 1993.

[8] G. Mar and P. Grim, "Pattern and chaos: New images in the semantics of paradox," No ǔs, vol. 25, pp. 659-694, 1991.

[9] — "Chaos, fractals, and the semantics of paradox," Res. Rep. 9101, Group for Logic and Formal Semantics, Dept. of Philos., SUNY at Stony Brook, 1991

[10] N. Rescher, Many-Valued Logic. New York: McGraw-Hill, 1969.

[11] L. A. Zadeh, "Fuzzy logic and approximate reasoning," Synthese, vol. 30, pp. $407-428,1975$.

[12] R. E. Bellman and M. Giertz, "On the analytic formalism of the theory of fuzzy sets," Inform. Sci., vol. 5, pp. 149-156, 1973

[13] Z. P. Dienes, "On an implication function in many-valued systems of logic," J. Symbol. Log., vol. 14, pp. 95-97, 1949.

[14] B. R. Gaines, "Foundations of fuzzy reasoning," Int. J. Man-Mach. Stud., vol. 8, pp. 623-668, 1976.
[15] L. A. Zadeh, "A fuzzy-set-theoretic interpretation of linguistic hedges," J. Cybernet., vol. 2, pp. 4-34, 1972.

[16] R. E. Bellman and L. A. Zadeh, "Local and fuzzy logics," in Modern Uses of Multiple-Valued Logic, J. Michael Dunn and George Epstein, Eds. Dordrecht: D. Reidel, 1977.

[17] J. F. Baldwin, "A new approach to approximate reasoning using a fuzzy logic," Fuzzy Sets and Syst., vol. 2, pp. 309-325, 1979.

[18] _ "Fuzzy logic and fuzzy reasoning," in Fuzzy Reasoning and its Appl., E. H. Mamdani and B. R. Gaines, Eds. New York: Academic, 1981.

[19] H.-J. Zimmerman, Fuzzy Set Theory and Its Applications. Dordrecht: Kluwer-Nijhoff, 1985.

[20] A. Tarski, "Der wahrheitsbegriff in den formalisierten sprachen," Studien Philosophica, vol. I, pp. 261-405, 1935.

[21] Y. Tsukamoto, "An approach to fuzzy reasoning method," in Advances in Fuzzy Set Theory and Applications, M. M. Gupta et. al, Eds. NorthHolland: Amsterdam, 1979, pp. 137-149.

[22] L. Ding, Z. Shen, and M. Mukaidono, "A new method for approximate reasoning," in Proc. 19th Int. Symp. on Multiple-Valued Logic. Washington, DC: IEEE Comput. Soc. Press, 1989, pp. 179-186.

[23] P. Grim, G. Mar, M. Neiger, and P. St. Denis, "Self-reference and paradox in two and three dimensions," Computers and Graphics.

[24] K. Gödel, "Über formal untscheidbare sätze der Principia Mathematica und verwandter systeme I, " Monatschefte für Mathematik und Physik, vol. 38, pp. 173-198, 1931.

[25] A. Church, "A note on the entscheidungsproblem," J. Symbol. Logic, vol. 1, pp. 40-41, 101-102, 1936.

[26] A. Turing, "On computable numbers, with an application to the entscheidungsproblem," in Proc. London Mathemat. Soc., 1936, vol. 42, pp. $230-265$.

[27] G. Chaitin, Information, Randomness, and Incompleteness--Papers on Algorithmic Information Theory. Singapore, World Scientific, 1990.

[28] B. van Fraassen, "Presupposition, implication, and self-reference," $J$. Philosophy, vol. 65, pp. 136-52, 1968.

[29] R. L. Martin, "A category solution to the liar," in The Paradox of the Liar, R. L. Martin, Ed. Reseda, CA: Ridgeview, 1970.

[30] S. Kripke, "Outline of a theory of truth," J. Philosophy, vol. 72, pp. $690-716,1975$.

[31] J. Barwise and J. Etchemendy, The Liar. New York: Oxford Univ. Press, 1987.

[32] J. L. Casti, Alternate Realities. New York: Wiley, 1989.

[33] G. Lakoff, Women, Fire, and Dangerous Things. Chicago, IL: Univ. of Chicago Press, 1987.

[34] N. Hellerstein, Isle of Paradox and Other Logic Adventures. 1992.

[35] C. Pickover, Computers and the Imagination. New York: St. Martin's, 1991.

[36] R. L. Devaney, An Introduction to Chaotic Dynamical Systems. Menlo Park, CA: Addison-Wesley, 1989.

[37] K. Falconer, Fractal Geometry: Mathematical Foundations and Applications. New York: Wiley, 1990.

[38] R. May, "Simple mathematical models with very complicated dynamics," Nature, vol. 261, pp. 459-467, 1976.

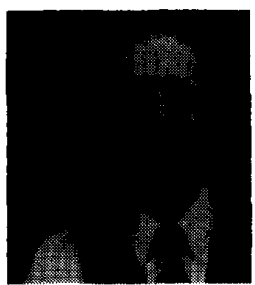

Patrick Grim received the A.B. degree in philosophy and anthropology from the University of California, Santa Cruz, in 1971, the B. Phil. degree from St. Andrews in 1975, and the A.M. and Ph.D. degrees in philosophy from Boston University in 1976.

He is author of The Incomplete Universe: Totality, Knowledge, and Truth (M.I.T. Press/Bradford Books, 1991) and co-editor of fourteen volumes of The Philosopher's Annual (Ridgeview Press). He is an Associate Professor and a member of the Group for Logic and Formal Semantics within the Department of Philosophy at the State University of New York at Stony Brook. His current research interests include alternative logics, game theory, dynamical systems, epistemic modeling, contemporary metaphysics, and ethics. 